\title{
Characterisations of Phase Transitions in Ising Spin Systems
}

\author{
M. Marinaro ${ }^{\star}$ and G. L. Sewell \\ Department of Physics, Queen Mary College, London, England
}

Received September 20, 1971

\begin{abstract}
We construct a $C^{*}$-algebraic formalism designed to provide a framework for the characterisation of phase transitions in a class of Ising spin systems: this class is large enough to include the rectangular lattice models, of arbitrary finite dimensionality, with nearest neighbour interactions. Using an extension of Onsager's transfer matrix formalism, we express properties of a Gibbs state of a system in terms of a contractive linear transformation, $v_{0}$, of a certain Hilbert space, the properties of $v_{0}$ being governed by the temperature as well as the interactions in the system. We obtain conditions on $v_{0}$ under which the system exhibits a phase transition characterised by (A) a thermodynamical singularity, (B) a change in symmetry, associated with the $G$-ergodic decomposition of Gibbs states, $(C)$ a divergence of a "correlation length" (appropriately defined) at the critical point, and (D) "scaling laws" in the critical region. Applying our formalism to the rectangular two-dimensional Ising model with nearest neighbour interactions, we show that its phase transition possesses the properties (B) and (C), as well as (A).
\end{abstract}

\section{Introduction}

The Ising model has for some time played a notable role in the theory of critical phenomena, due to the fact that it provides a relatively simple example of a phase transition of the second type which does not correspond to a mean field theory. In particular, the two-dimensional version of the model, with nearest neighbour interactions, $\mathscr{J}_{2}$, has proved to be exactly solvable in certain respects, and thus has provided a testingground for various general ideas concerning critical phenomena. Specifically, Onsager [1] has obtained an exact solution for the thermodynamical functions of $\mathscr{J}_{2}$, demonstrating that its specific heat has a singularity at a well-defined temperature $\tau_{c}$. Among the significant extensions of Onsager's solution is the treatment by Montroll, Potts and Ward [2] of the correlations between a pair of spins in $\mathscr{J}_{2}$, revealing a transition from short-range order (clustering) to long-range order (non-clustering) on passing through $\tau_{c}$ from above. On a heuristic level, Kadanoff and collaborators [3] have based a theory of the so-called "scaling laws" on the behaviour of these correlations in the critical region.

\footnotetext{
* Permanent address: Istituto di Fisica Teorica, Università di Napoli, Napoli, Italy.
} 
The object of the present article is to provide a $C^{*}$-algebraic framework for the characterisation of critical phenomena in a certain class of Ising spin systems: this class is large enough to include all finite dimensional rectangular lattice models with nearest neighbour interactions. Our main aim is to obtain conditions under which a given Ising system exhibits a phase transition characterised by:

(A) a singularity in a thermodynamical potential at a temperature $\tau_{c}$;

(B) a spontaneous symmetry change ${ }^{1}$, associated with a spatiallyergodic decomposition of the Gibbs state, on passing through $\tau_{c}$;

(C) the divergence of a "correlation length" (suitably defined), as the temperature $\tau \rightarrow \tau_{c} \pm 0$; and

(D) scaling laws in the neighbourhood of $\tau_{c}$.

Our investigation is centred on the infinite-volume Gibbs states of the system. By extending Onsager's transfer matrix method, we are able, to express properties of such a state in terms of a contractive transformation, $v_{0}$, of a certain Hilbert space ${ }^{1 \mathrm{a}}$. The properties of $v_{0}$ are determined by the temperature and by the interactions in the system. Thus, we obtain conditions on $v_{0}$, i.e. on the temperature and interactions, under which the system exhibits a phase transition, with properties (A)-(D). In the particular case of $\mathscr{J}_{2}$, we show that the transition is characterised by (B) and (C), as well as (A).

The subject-matter of the article will be presented as follows. In Section 2, we formulate the $C^{*}$-algebra of observables, $\mathscr{A}$, for a system of Ising spins on an assembly of sites, $S=T \times Z$, where $Z$ is the set of integers and $T$ is an arbitrary countably infinite point set. We define automorphisms $\varrho, G$ of $\mathscr{A}$, where $\varrho$ corresponds to spin reversals and $G$ to the group of space translations along the $Z$ component of $T \times Z$. We also define auxilliary $C^{*}$-algebras $\mathscr{B}, \hat{\mathscr{B}}$ generated respectively by Ising and Pauli spins on the set $T$.

In Section 3, we specify the interactions between the spins, and then construct the Gibbs state, $\phi$, on $\mathscr{A}$, for temperature $\tau$, as a limit of finite volume Gibbs states.

In Section 4, we employ the transfer matrix formalism to express the properties of $\phi$ in terms of those of an associated state $\Psi$ on $\hat{B}$. This leads to a formulation of the $G$-correlation functions, for $\phi$, in terms of a semigroup, $\left\{v_{0}^{n} \mid n \in Z_{+}\right\}$, of contractions of a certain subspace, $\mathfrak{H}_{0}$, of the

${ }^{1}$ A different kind of symmetry change has been formulated by Dobrushin [4], who has shown that, in certain cases, the state of an Ising spin system can depend on symmetrybreaking boundary conditions, even in the thermodynamical limit.

1a After this manuscript was completed, D. Ruelle kindly drew our attention to a recent paper by R. A. Minlos and Ja. G. Sinai (Teor. i Matem. Fizika 2, (2), 230-243 (1970)) in which certain properties of Ising spin system are expressed in terms of a stochastic operator $P_{\infty}$. It may readily be seen that this latter operator is closely related to the one denoted by $v_{0}$ in the present article. 
GNS space for $\Psi$ (Proposition 4.1). Thus we obtain conditions on $v_{0}$ for the $G$-ergodicity, and for spontaneous $\varrho$-symmetry breakdown of the state $\phi$ associated with its $G$-ergodic decomposition (Propositions 4.2, 4.3).

In Section 5, we employ the results of Section 4 to specify conditions under which the system exhibits a phase transition characterised by (A), (B) and (C). In particular, we show that $\mathscr{J}_{2}$ exhibits a transition which it is characterised by these properties.

In Section 6, we obtain scaling laws for the critical region, on the basis of the results of Section 4, supplemented by additional assumptions on the spectral properties of the contraction $v_{0}$. In Section 7 , we summarise our conclusions.

Throughout the article, we shall use the standard symbols $R, R_{+}$, $Z, Z_{+}$and $C$ to denote the real line, the positive reals, the integers, the non-negative integers and the complex plane respectively. If $a, b \in Z$, we denote by $[a, b]$ the set $\{n \mid n \in Z ; a \leqq n \leqq b\}$. If $\mathscr{H}$ is a Hilbert space, we shall denote by $\mathscr{L}(\mathscr{H})$ the set of all bounded operators in $\mathscr{H}$. If $E$ is a topological space, we shall denote by $\mathscr{C}(E)$ the set of all complex-valued continuous functions on $E$. If $F$ is a countable set, we denote by $\mathscr{P}(F)$ (resp. $\left.\mathscr{P}_{f}(F)\right)$ the class of all (resp. finite) subsets of $F$.

\section{The Observables}

Let $S$, the assembly of sites, be a set $T \times Z$, where $T$ is a countably infinite point set. Denoting points in $T, Z, S$ by $t, n, s=(t, n)$, respectively, we define $\left\{u^{n} \mid n \in Z\right\}$ to be the group of transformations of $S$ given by

$$
u^{n}\left(t, n^{\prime}\right)=\left(t, n+n^{\prime}\right), \forall t \in T ; n, n^{\prime} \in Z .
$$

For $n \in Z$ and $L \in \mathscr{P}(S)$, we define $u^{n} L=\left\{s \mid u_{-n} s \in L\right\}$.

Let $K$ be the set $\{-1,1\}$, equipped with the discrete topology: $K$ thus corresponds to the set of possible values of an Ising spin at a site. For $L \in \mathscr{P}(S), M \in \mathscr{P}(T)$, we define $X_{L}, Y_{M}$ to be the topological powers $K^{L}, K^{M}$, respectively. Thus, $X_{L}$ (resp. $Y_{M}$ ) is the spin configuration space for $L$ (resp. $M$ ). It follows from our definitions of these spaces that $X_{L}$, $Y_{M}$ are compact (Tychonoff's theorem) and that

and

$$
\begin{aligned}
& X_{L \cup L^{\prime}}=X_{L} \times X_{L^{\prime}} \quad \text { for } \quad L, L^{\prime} \in \mathscr{P}(S), L \cap L^{\prime}=\emptyset, \\
& Y_{M \cup M^{\prime}}=Y_{M} \times Y_{M^{\prime}} \quad \text { for } \quad M, M^{\prime} \in \mathscr{P}(T), M \cap M^{\prime}=\emptyset \text {, } \\
& X_{M \times N}=Y_{M}^{N} \quad \text { for } \quad M \in \mathscr{P}(T), N \in \mathscr{P}(Z) \text {. }
\end{aligned}
$$

Elements of $X_{L}, Y_{M}$ will be denoted by $x_{L}, y_{M}$, respectively. For $s \in L$ (resp. $t \in M$ ), we shall denote the $s^{\prime}$ th projection of $x_{L}$ (resp. $t^{\prime}$ th projection 
of $y_{M}$ ) by $x_{s}$ (resp. $y_{t}$ ). We shall sometimes denote $x_{M \times N}$ by $y_{M}^{N}$, and, for $n \in N$, we shall denote the $n^{\prime}$ th projection of $y_{M}^{N}$ by $y_{M}^{(n)}$.

We define $\mathscr{A}$ (resp. $\mathscr{B})$ to be the $C^{*}$-algebra $\mathscr{C}\left(X_{S}\right)$ (resp. $\mathscr{C}\left(Y_{T}\right)$ ), equipped with the uniform norm. $\mathscr{A}$ is taken to be the algebra of observables for the Ising spin system. For $L \in \mathscr{P}_{f}(S)$ (resp. $M \in \mathscr{P}_{f}(T)$ ), we define $\mathscr{A}_{L}$ (resp. $\mathscr{B}_{M}$ ) to be the subalgebra of $\mathscr{A}$ (resp. $\mathscr{B}$ ) whose elements $A$ (resp. $B$ ) possess the property that, if $x_{S}=\left(x_{L}, x_{S \backslash L}\right)\left(\right.$ resp. $\left.y_{T}=\left(y_{M}, y_{T \backslash M}\right)\right)$ then $A\left(x_{S}\right)$ (resp. $\left.B\left(y_{T}\right)\right)$ is independent of $x_{S \backslash L}$ (resp. $y_{T \backslash M}$ ). Hence $\mathscr{A}_{L}$ (resp. $\mathscr{B}_{M}$ ) is isotonic with respect to $L$ (resp. M). Defining $\mathscr{A}_{\mathscr{S}}=\bigcup_{L \in \mathscr{\mathscr { P }}_{f}(S)} \mathscr{A}_{L}$ and $\mathscr{B}_{\mathscr{I}}=\bigcup_{M \in \mathscr{P}_{f}(T)} \mathscr{B}_{M}$, it follows from the Stone-Weierstrass theorem that $\mathscr{A}_{\mathscr{S}}, \mathscr{B}_{\mathscr{I}}$ are dense in $\mathscr{A}, \mathscr{B}$, respectively.

Definition 2.1. For $L \in \mathscr{P}_{f}(S), M \in \mathscr{P}_{f}(T)$ we define the bijective maps $p_{L}: \mathscr{A}_{L} \rightarrow \mathscr{C}\left(X_{L}\right)$ and $q_{M}: \mathscr{B}_{M} \rightarrow \mathscr{C}\left(Y_{M}\right)$ by the formulae

$p_{L} A=A_{L} ; A_{L}\left(x_{L}\right)=A\left(x_{L}, x_{S \backslash L}\right) ; \forall A \in \mathscr{A}_{L}, x_{L} \in X_{L}, x_{S \backslash L} \in X_{S \backslash L}$ and

$q_{M} B=B_{M} ; B_{M}\left(y_{M}\right)=B\left(y_{M}, y_{T \backslash M}\right) ; \forall B \in \mathscr{B}_{M}, y_{M} \in Y_{M}, y_{T \backslash M} \in Y_{T \backslash M}$.

Note. These definitions are unambiguous because of the independence of $A\left(x_{L}, x_{S \backslash L}\right)$ (resp. $B\left(y_{M}, y_{T \backslash M}\right)$ ) of $x_{S \backslash L}$ (resp. $y_{T \backslash M}$ ) for $A \in \mathscr{A}_{L}$ (resp. $B \in \mathscr{B}_{M}$ ).

Definition 2.2. For $M \in \mathscr{P}_{f}(T), N \in \mathscr{P}_{f}(Z)$, we define $\tilde{\mathscr{A}}_{M N}$ to be the subset of $\mathscr{A}_{M \times N}$ whose elements $A$ are of the form

$$
A(x)=\prod_{n \in N} B^{(n)}\left(y^{(n)}\right),
$$

where the $B^{(n)}$ 's $\in \mathscr{B}_{M} \backslash I_{M}$. We shall denote $A$ by $\prod_{n \in N} B^{(n)}$. Further, we
define $\tilde{\mathscr{A}}=U \tilde{\mathscr{A}}_{M N}$. define $\tilde{\mathscr{A}}=\bigcup_{\substack{M \in \mathscr{P}_{f}(T) \\ N \in \mathscr{P}_{f}(\mathrm{Z})}} \tilde{\mathscr{M}}_{M N}$.

Note. It follows easily from Definition 2.2 and the Stone-Weierstrass theorem that, for $M \in \mathscr{P}_{f}(T)$ and $N \in \mathscr{P}_{f}(Z), \mathscr{A}_{M \times N}$ is the $C^{*}$-algebra generated by $\tilde{\mathscr{A}}_{M N}$, and is also the norm closure of the space of finite complex linear combinations of elements of $\tilde{\mathscr{A}}_{M N}$. Hence, $\mathscr{A}$ is the norm closure of the set of finite complex linear combinations of elements of $\tilde{\mathscr{A}}$, i.e., $\tilde{\mathscr{A}}$ serves as a basis set for $\mathscr{A}$.

Definition 2.3. (i) We define $G$ (resp. $G_{+}$) to be the group (resp. semigroup) $\left\{\tau_{n} \mid n \in Z\left(\right.\right.$ resp. $\left.\left.Z_{+}\right)\right\}$of automorphisms of $\mathscr{A}$ given by

where

$$
\left(\tau_{n} A\right)(x)=A\left(\tau_{n}^{\prime} x\right), \quad \forall x \in X_{S}, \quad n \in Z
$$

Thus, $G$ corresponds to space translations along the $Z$-component of $S$. 
(ii) We define $\varrho(\in$ Aut $\mathscr{A})$ by the formula

$$
(\varrho A)(x)=A\left(\varrho^{\prime} x\right), \quad \forall A \in \mathscr{A}, \quad x \in X_{S}
$$

where $\left(\varrho^{\prime} x\right)_{s}=-x_{s}, \forall x \in X_{S}, s \in S$.

Thus, $\varrho$ corresponds to spin reversals.

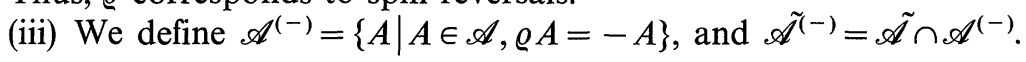

Definition 2.4. (i) We define $\mathfrak{G}_{G}$ to be the set of states, $\psi$, on $\mathscr{A}$ which are invariant with respect to $G$, i.e. $\psi=\psi \circ \tau_{n}, \forall n \in Z$.

(ii) We define $\mathscr{E}_{G}$ to be the set of $G$-ergodic states on $\mathscr{A}$, i.e. $\mathscr{E}_{G}$ is the set of extremal elements of the convex, $w^{*}$-compact set $\mathfrak{G}_{G}$.

(iii) We define $\mathfrak{b}_{\varrho}$ to be the set of states, $\psi^{\prime}$, on $\mathscr{A}$ that are invariant with respect to $\varrho$, i.e. $\psi^{\prime}=\psi^{\prime} \circ \varrho$.

Having defined $\mathscr{A}$ and $\mathscr{B}$, we now introduce another algebra, $\hat{\mathscr{B}}$, as follows. For each $M \in \mathscr{P}_{f}(T)$, we define $\mathscr{H}_{M}$ to be the finite-dimensional Hilbert space

$$
\left\{f \mid f \in \mathscr{C}\left(Y_{M}\right) ;(f, g)_{\mathscr{H}_{M}}=\sum_{y_{M} \in Y_{M}} \bar{f}\left(y_{M}\right) g\left(y_{M}\right)\right\} .
$$

It follows from this definition and Eq. (2.3) that

$$
\mathscr{H}_{M \cup M^{\prime}}=\mathscr{H}_{M} \otimes \mathscr{H}_{M^{\prime}} ; \quad \forall M, M^{\prime} \in \mathscr{P}_{f}(T), \quad M \cap M^{\prime}=\emptyset .
$$

For $M \in \mathscr{P}_{f}(T)$, let $\hat{\mathscr{B}}_{M}$ denote the algebra of bounded operators in $\mathscr{H}_{M}$, equipped with the uniform norm. For $M \subset M^{\prime} \in \mathscr{P}_{f}(T)$, we identify $\hat{B}\left(\in \hat{\mathscr{B}}_{M}\right)$ with $\hat{B} \otimes I_{M^{\prime} \backslash M}\left(\in \hat{\mathscr{B}}_{M^{\prime}}\right)$. Under this identification, $\hat{\mathscr{B}}_{M}$ is isotonic with respect to $M$. We define $\mathscr{B}$ to be the $C^{*}$-algebra given by the norm completion of $\bigcup_{M \in \mathscr{P}_{f}(T)} \hat{\mathscr{B}}_{M}\left(\equiv \hat{\mathscr{B}}_{\mathscr{I}}\right)$. It follows from our definitions that $\hat{\mathscr{B}}$ possesses the local commutivity property:

$$
\hat{\mathscr{B}}_{M_{1}} \in \hat{\mathscr{B}}_{M_{2}}^{\prime} \text {, for } \quad M_{1}, M_{2} \in \mathscr{P}_{f}(T) \text {, with } \quad M_{1} \cap M_{2}=\emptyset \text {. }
$$

Definition 2.5. (i) For $M \in \mathscr{P}_{f}(T)$, we define $\alpha_{M}$ to be the bijective map of $\hat{\mathscr{B}}$ onto $\mathscr{C}\left(Y_{M} \times Y_{M}\right)$ given by:

$$
\alpha_{M} \hat{B}=B_{M} ;(\hat{B} f)\left(y_{M}\right)=\sum_{y_{M}^{\prime} \in Y_{M}} B_{M}\left(y_{M}, y_{M}^{\prime}\right) f\left(y_{M}^{\prime}\right) ; \forall f \in \mathscr{H}_{M}, y_{M} \in Y_{M} \text {. }
$$

(ii) For $t \in T$ and $r=1,2,3$, we define $\hat{\sigma}_{t}^{(r)} \in \hat{\mathscr{B}}_{\{t\}}$ by:

$$
\left(\alpha_{\{t\}} \hat{\sigma}_{t}^{(1)}\right)\left(y_{t}, y_{t^{\prime}}\right)=\frac{1}{2}\left(y_{t}+y_{t^{\prime}}\right) ; \quad\left(\alpha_{\{t} \hat{\sigma}_{t}^{(2)}\right)\left(y_{t}, y_{t}^{\prime}\right)=\frac{i}{2}\left(y_{t}-y_{t}^{\prime}\right) ;
$$

and $\left(\alpha_{\{t} \hat{\sigma}_{t}^{(3)}\right)\left(y_{t}, y_{t^{\prime}}\right)=\frac{1}{2}\left(1-y_{t} y_{t}^{\prime}\right)$. 
(iii) For $M \in \mathscr{P}_{f}(T)$ and $r=1,2,3$, we define $\hat{\sigma}_{M}^{(r)}$ by the formula

$$
\hat{\sigma}_{M}^{(r)}=\sum_{t \in M} \hat{\sigma}_{t}^{(r)} .
$$

Note. It follows from Definition 2.5 that the $\hat{\sigma}_{t}^{(r)}$ 's conform to the algebra of Pauli matrices, i.e.

$\left[\hat{\sigma}_{t}^{(r)}, \hat{\sigma}_{t^{\prime}}^{\left(r^{\prime}\right)}\right]_{-}=0$ for $t \neq t^{\prime} ;$ and

$\left.\left(\hat{\sigma}_{t}^{(r)}\right)^{2}=I ; \quad \hat{\sigma}_{t}^{(1)} \hat{\sigma}_{t}^{(2)}=i \hat{\sigma}_{t}^{(3)} ; \quad \hat{\sigma}_{t}^{(3)} \hat{\sigma}_{t}^{(1)}=i \hat{\sigma}_{t}^{(2)} ; \quad \hat{\sigma}_{t}^{(2)} \hat{\sigma}_{t}^{(3)}=i \hat{\sigma}_{t}^{(1)}.\right\}$

It also follows from our definitions of $\hat{\mathscr{B}}$ and the $\hat{\sigma}$ 's that $\hat{\mathscr{B}}$ is the $C^{*}$ algebra generated by $\left\{\hat{\sigma}_{t}^{(r)} \mid t \in T ; r=1,2,3\right\}$.

Definition 2.6. For $M \in \mathscr{P}_{f}(T)$, we define $\delta_{M}$ to be the imbedding of $\mathscr{B}_{M}$ into $\hat{\mathscr{B}}_{M}$ given by:

$\left(\left(\delta_{M} B\right) f\right)\left(y_{M}\right)=\left(q_{M} B\right)\left(y_{M}\right) f\left(y_{M}\right) ; \forall B \in \mathscr{B}_{M}, f \in \mathscr{H}_{M}, y_{M} \in Y_{M}$.

We define $\delta$ to be the imbedding of $\mathscr{B}$ into $\hat{\mathscr{B}}$ given by

$$
\delta_{\mid \mathscr{B}_{M}}=\delta_{M}, \quad \forall M \in \mathscr{P}_{f}(T) .
$$

We shall denote the image of $\mathscr{B}$ (resp. $\mathscr{B}_{M}$ ) under $\delta$ by $\hat{\mathscr{B}}^{(0)}\left(\right.$ resp. $\left.\hat{\mathscr{B}}_{M}^{(0)}\right)$.

\section{Interactions and Gibbs States}

We shall formulate the properties of the infinite assembly of spins in terms of those of a sequence of finite sub-assemblies. For this purpose, we introduce covers $\mathscr{M}\left(\in \mathscr{P}_{f}(T)\right)$ and $\mathscr{N}\left(\in \mathscr{P}_{f}(Z)\right)$ of $T$ and $Z$, respectively, where $\mathcal{N}=\{[a, b] ; a, b(>a) \in Z\}$. We assign to each $M \times N(M \in \mathscr{M}$, $N \in \mathscr{N})$ a Hamiltonian $H_{M N}$ (a real-valued element of $\left.\mathscr{C}\left(Y_{M}^{N}\right)\right)$ corresponding to the potential energy of a system of spins confined to that region. It is assumed that $H_{M N}$ is of the form

$$
H_{M N}\left(y_{M}^{N}\right)=\sum_{n \in N} f_{M}\left(y_{M}^{(n)}\right)+\sum_{n \in N} g_{M}\left(y_{M}^{(n)}, y_{M}^{(n+1)}\right),
$$

where, for $N=[a, b],(b+1)$ is identified with $a$ in the last sum (cyclical boundary conditions). Clearly, $f_{M}\left(y_{M}^{(n)}\right)$ corresponds to the energy of interaction between spins in the "hyperplane" $T \times\{n\}$, and $g_{M}\left(y_{M}^{(n)}\right.$, $y_{M}^{(n+1)}$ ) represents the interaction between neighbouring hyperplanes. We shall assume that $g_{M}$ is of the form

$$
g_{M}\left(y_{M}, y_{M}^{\prime}\right)=-J \sum_{t \in M} y_{t} y_{t}^{\prime}
$$

with $J \in R_{+}$; and that $f_{M}$ possesses the following property $(P)$ : There exists a map $v: \mathscr{M} \rightarrow \mathscr{P}_{f}(T)$ such that, if $M^{\prime} \in \mathscr{M}$ and $v\left(M^{\prime}\right) \subset M \in \mathscr{M}$, 
then $f_{M}$ may be expressed in the form

$$
f_{M}\left(y_{M}\right)=f_{v\left(M^{\prime}\right)}^{(1)}\left(y_{v\left(M^{\prime}\right)}\right)+f_{M \backslash M^{\prime}}^{(2)}\left(y_{M \backslash M^{\prime}}\right)
$$

where

$$
f_{v\left(M^{\prime}\right)}^{(1)} \in \mathscr{C}\left(y_{v\left(M^{\prime}\right)}\right) \text {, and } f_{M \backslash M^{\prime}}^{(2)} \in \mathscr{C}\left(y_{M \backslash M^{\prime}}\right) .
$$

Physically, $v\left(M^{\prime}\right)$ corresponds to the union of $M^{\prime}$ with the maximal subset of $T \backslash M^{\prime}$ whose spins are coupled to those in $M^{\prime}$. Thus, (P) signifies that the interactions within each hyperplane are of finite range.

The theory will be concerned specifically with the following cases.

Case I. This is $\mathscr{J}_{2}$, the two-dimensional Ising model, with nearest neighbour interactions and toroidal boundary conditions, in the absence of any external magnetic field. In this case, $T=Z, \mathscr{M}$ is taken to be $\left\{\left[a^{\prime}, b^{\prime}\right] ; a^{\prime}, b^{\prime}\left(>a^{\prime}\right) \in Z\right\}$, and $f_{M}$ is given by the formula

$$
f_{M}\left(y_{M}\right)=-J^{\prime} \sum_{t \in M} y_{t} y_{t+1}
$$

where $J^{\prime} \in R_{+}$and, for $M=\left[a^{\prime}, b^{\prime}\right],\left(b^{\prime}+1\right)$ is identified with $a^{\prime}$.

Case II. In this case, $T$ is an arbitrary, countably infinite point set, $\mathscr{M}=\mathscr{P}_{f}(T)$, and $f_{M}$ corresponds to interactions between finite groups of spins in $M$. Specifically, there exists a positive integer $p_{0}$ and, for $p \in\left[1, p_{0}\right]$, a non-negative function $J^{(p)}$ on $T^{p}$ such that

$$
f_{M}\left(y_{M}\right)=-\sum_{p \in\left[1, p_{0}\right]} \sum_{t_{1}, \ldots, t_{p} \in M} J^{(p)}\left(t_{1}, \ldots, t_{p}\right) y_{t_{1}} \ldots y_{t_{p}} .
$$

Further, the functions $J^{(p)}$ are stipulated to accord with property (P): i.e., given $p \in\left[1, p_{0}\right]$ and $t \in T$, there exists at most a finite set of points $t^{\prime}$, in $T$, such that $\left\{t, t^{\prime}\right\} \subset$ a set $\left\{t_{1}, \ldots, t_{p}\right\}$ for which $J^{(p)}\left(t_{1}, \ldots, t_{p}\right) \neq 0$.

Case $I I^{\prime}$. This corresponds to Case II, with the restriction that $J^{(p)}=0$ when $p$ is odd.

Note. II includes the cases of rectangular Ising models of arbitrary finite dimensionality $d$ (i.e. $T=Z^{d-1}$ ) with finite range interactions in each hyperplane $T \times\{n\}$, in the presence of an external magnetic field (represented by $\left.J^{(1)}\right)$.

Note. The Case $\mathrm{II}^{\prime}$ corresponds to the restriction of II to cases where $H_{M N}$ is invariant under spin reversals $\left(y_{M} \rightarrow-y_{M}\right)$, and thus where there is no external magnetic field.

Definition 3.1. For $M \in \mathscr{M}, N \in \mathscr{N}$, we define the Gibbs state $\phi_{M N}$, on $\mathscr{A}_{M \times N}$, corresponding to the inverse temperature $\beta$, by the formulae

$$
\phi_{M N}(A)=E_{M N}(A) / E_{M N}(I)
$$

with

$$
E_{M N}(A)=\sum_{y_{M}^{N} \in Y_{M}^{N}} A_{M \times N}\left(y_{M}^{N}\right) \exp \left[-\beta H_{M N}\left(y_{M}^{N}\right)\right]
$$

and

$$
A_{M \times N}=p_{M \times N} A .
$$


The following lemma, concerning the properties of $\phi_{M N}$ in the thermodynamical limit, will be derived in Appendix 1 from standard properties of the Ising model.

Lemma 3.1. (i) In both cases (I) and (II), there exists a unique state $\phi$ on $\mathscr{A}$, such that

$$
\phi(A)=\mathscr{M}-\lim _{M \rightarrow \infty} \mathscr{N}-\lim _{N \rightarrow \infty} \phi_{M N}(A), \quad \forall A \in \mathscr{A}_{\mathscr{S}}
$$

where $\mathscr{M}-\lim _{M \rightarrow \infty}\left(\right.$ resp. $\left.\mathscr{N}-\lim _{N \rightarrow \infty}\right)$ refers to the limit with respect to any increasing sequence of elements of $\mathscr{M}$ (resp. $\mathcal{N}$ ) whose union covers $T$ (resp. Z).

We shall refer to $\phi$ as the Gibbs state corresponding to the inverse temperature $\beta$.

(ii) $\phi \in \mathfrak{G}_{G}$.

(iii) In Cases (I) and (II'), $\phi \in \mathfrak{6}_{\varrho^{\circ}}$.

Definition 3.2. (i) We define $\mu$ to be the (unique) measure ${ }^{2}$ on $\mathscr{E}_{G}$ induced by the $G$-ergodic decomposition of $\phi$, i.e.

$$
\phi=\int_{\mathscr{E}_{G}} \phi_{\alpha} d \mu(\alpha) .
$$

(ii) We say that $\phi$ undergoes a $\varrho$-symmetry breakdown associated with its $G$-ergodic decomposition if $\phi \in \mathfrak{b}_{\varrho}$ and $\mu\left(\mathscr{E}_{G} \backslash \mathfrak{b}_{\varrho}\right) \neq 0$.

Definition 3.3. (i) We define $F: \mathscr{A} \times \mathscr{A} \times Z \rightarrow C$ by the formula

$$
F\left(A^{\prime}, A: n\right)=\phi\left(A^{\prime} \tau_{n} A\right) ; \forall A^{\prime}, A \in \mathscr{A}, \quad n \in Z .
$$

(ii) We define $F_{\eta}: \mathscr{A} \times \mathscr{A} \rightarrow C$ to be the ergodic mean ${ }^{3}$ of $F$, i.e.

$$
F_{\eta}\left(A^{\prime}, A\right)=\lim _{m \rightarrow \infty} \frac{1}{m} \sum_{n=1}^{m} F\left(A^{\prime}, A ; n\right) ; \quad \forall A^{\prime}, A \in \mathscr{A} .
$$

(iii) we define $F^{\prime}: \mathscr{A} \times \mathscr{A} \times Z \rightarrow C$ by the formula

$$
F^{\prime}\left(A^{\prime}, A ; n\right)=F\left(A^{\prime}, A ; n\right)-F_{\eta}\left(A^{\prime}, A\right) ; \forall A^{\prime}, A \in \mathscr{A}, n \in Z \text {. }
$$

Note. Since $\mathscr{A}$ is Abelian, it follows from Lemma 3.1 (ii) and Definition 3.3 (i) that $F\left(A^{\prime}, A ; n\right) \equiv F\left(A, A^{\prime} ;-n\right)$, and thus $F$ is determined by its restriction to $\mathscr{A} \times \mathscr{A} \times Z_{+}$. Consequently, since $\mathscr{A}$ is a basis set in $\mathscr{A}$, it follows that $F$ is determined by its restriction to $\tilde{\mathscr{A}} \times \tilde{\mathscr{A}} \times Z_{+}$. Likewise

${ }^{2}$ The uniqueness of the $G$-ergodic decomposition in the present case follows easily from the general treatment of $G$-Abelian groups, by Lanford and Ruelle [5].

3 The existence of this mean follows easily from the application of Riesz's ergodic theorem (Ref. [8], p. 407) to the unitary representation of $Z$ induced by $G$ in the $G N S$ space for $\phi$.

23 Commun. math. Phys., Vol. 24 
$F^{\prime}, F_{\eta}$ are determined by their restrictions to $\tilde{\mathscr{A}} \times \tilde{\mathscr{A}} \times Z_{+}, \tilde{\mathscr{A}} \times \tilde{\mathscr{A}}$, respectively.

Note. Since the elements of $\mathscr{E}_{G}$ are weakly $G$-clustering, it follows from Definitions 3.2 (ii), 3.3 (iii) that

$$
F_{\eta}\left(A^{\prime}, A\right)=\int_{\mathscr{E}_{G}} d \mu(\alpha) \phi_{\alpha}\left(A^{\prime}\right) \phi_{\alpha}(A), \quad \forall A^{\prime}, A \in \mathscr{A} ;
$$

and

$$
F^{\prime}\left(A^{\prime}, A ; n\right)=\int_{\mathscr{E}_{G}} d \mu(\alpha)\left[\phi_{\alpha}\left(A^{\prime} \tau_{n} A\right)-\phi_{\alpha}\left(A^{\prime}\right) \phi_{\alpha}(A)\right] ; \forall A^{\prime}, A \in \mathscr{A} ; n \in Z \text {. }
$$

Since the integrand in (3.15) corresponds to the $G$-correlation function for the ergodic state $\phi_{\alpha}$, we shall refer to $F^{\prime}$ for the $\mu$-correlation function for $\phi$.

Lemma 3.2. If $F_{\eta}$ does not vanish on $\mathscr{A}^{(-)} \times \mathscr{A}^{(-)}$, and if $\phi \in \mathfrak{G}_{\varrho}$, then $\phi$ undergoes a @-symmetry breakdown associated with its G-ergodic decomposition.

Proof. Assume that $F_{\eta}$ does not vanish on $\mathscr{A}^{(-)} \times \mathscr{A}^{(-)}$. Then it follows from Eq. (3.14) that $\exists A^{\prime}, A \in \mathscr{A}^{(-)}$such that $\int_{\mathscr{E}_{G}} d \mu(\alpha) \phi_{\alpha}\left(A^{\prime}\right) \phi_{\alpha}(A)$ $\neq 0$. Hence, $\phi_{\alpha \mid \mathscr{A}(-)}$ cannot vanish a.e. on $\operatorname{supp} \mu$; and consequently, by Definitions 2.3 (iii), 2.4 (iii), $\phi_{\alpha}$ cannot belong to $\mathfrak{b}_{\varrho}$ a.e. on $\operatorname{supp} \mu$. Therefore, $\mu\left(\mathscr{E}_{G} \backslash\left(\mathfrak{G}_{e}\right) \neq 0\right.$, which proves the lemma.

Q.E.D.

\section{The Transfer Matrix Formalism}

In this Section, we present a formalism which enables us to express the $G$-correlation functions for the state $\phi$ in terms of a one-parameter semi-group of contractions of a certain Hilbert space (Propositions 4.1, 4.2).

Definition 4.1. Corresponding to the above formulation of $H_{M N}$ we define $V_{M} \in \mathscr{C}\left(Y_{M} \times Y_{M}\right)$ (for $\left.M \in \mathscr{M}\right)$ by the formula

$$
V_{M}\left(y_{M}, y_{M}^{\prime}\right)=\exp \left(-\frac{1}{2} \beta f_{M}\left(y_{M}\right)\right) \exp \left(-\beta J \sum_{t \in M} y_{t} y_{t}^{\prime}\right) \exp \left(-\frac{1}{2} \beta f_{M}\left(y_{M}^{\prime}\right)\right) \text {. }
$$

We then define $\hat{V}_{M}\left(\in \hat{\mathscr{B}}_{M}\right)$ and $\hat{f}_{M}\left(\in \hat{\mathscr{B}}_{M}^{(0)}\right)$ by the formulae

$$
\hat{V}_{M}=\alpha_{M} V_{M}
$$

and

$$
\hat{f}_{M}=\delta_{M} f_{M}
$$

i.e., by Definitions 2.5 and 2.6

$$
\hat{V}_{M}=C_{M} \exp \left(-\frac{1}{2} \beta \hat{f}_{M}\right) \exp \left(J_{\beta}^{*} \hat{\sigma}_{M}^{(3)}\right) \exp \left(-\frac{1}{2} \beta \hat{f}_{M}\right)
$$

where

$$
J_{\beta}^{*}=\tanh ^{-1}\left(2 e^{-\beta J}\right)
$$


and $C_{M}=\left(2 \sinh 2 J_{\beta}^{*}\right)^{\frac{1}{2} \gamma_{M}}$, where $\gamma_{M}$ is the number of points in $M$. In accordance with a usual terminology, we shall refer to $\hat{V}_{M}$ as the transfer matrix.

Note. It follows from Eq. (4.4) that $\hat{V}_{M}$ is a positive selfadjoint bounded, invertible operator in the finite-dimensional Hilbert space $\mathscr{H}_{M^{\prime}}$. Further, by Eq. (4.1), $V_{M}\left(y_{M}, y_{M^{\prime}}\right)$ in positive and therefore, by Frobenius's theorem, the principal (i.e. largest) eigenvalue of $\hat{V}_{M}$ is non-degenerate.

Definition 4.2. Let the principal (normalised) eigenvector and eigenvalue of $\hat{V}_{M}$ be $\hat{\Omega}_{M}, \Lambda_{M}$, respectively. Then:

(i) We define $\hat{v}_{M}$, the normalised transfer matrix, by the formula

$$
\hat{v}_{M}=\hat{V}_{M} / \Lambda_{M}
$$

and we denote by $\left\{\hat{E}_{M}(\lambda)\right\}$ the family of spectral projectors for $\hat{v}_{M}$ $\left(\hat{v}_{M}=\int \lambda d \hat{E}_{M}(\lambda)\right)$.

(ii) We define $w_{M}: \hat{\mathscr{B}}_{M} \rightarrow \hat{\mathscr{B}}_{M}$ by the formula

$$
w_{M} \hat{B}=\hat{v}_{M} \hat{B} \hat{v}_{M}^{-1}\left(\equiv \hat{V}_{M} \hat{B} \hat{V}_{M}^{-1}\right), \quad \forall \hat{B} \in \hat{\mathscr{B}}_{M} .
$$

(iii) We define $\Psi_{M}$ to be the state on $\hat{\mathscr{B}}_{M}$ given by:

$$
\Psi_{M}(\hat{B})=\left(\hat{\Omega}_{M}, \hat{B} \hat{\Omega}_{M}\right), \quad \forall \hat{B} \in \hat{\mathscr{B}}_{M} .
$$

(iv) We introduce a cover $\mathscr{M}^{\prime}(\subset \mathscr{M})$ of $T$ such that $\mathscr{M}^{\prime}-\lim _{M \rightarrow \infty} \Psi_{M}(B)$ exists, $\forall \hat{B} \in \hat{\mathscr{B}}_{\mathscr{I}}$ : such a cover may be constructed by means of the diagonal process, in view of the fact that $\hat{\mathscr{B}}_{M}$ is a finite-dimensional vector space when $M \in \mathscr{P}_{f}(T)$. We define $\Psi$ to be the unique ${ }^{4}$ state on $\mathscr{B}$ for which

$$
\Psi(\hat{B})=\mathscr{M}^{\prime}-\lim _{M \rightarrow \infty} \Psi_{M}(\hat{B}), \quad \forall \hat{B} \in \hat{\mathscr{B}}_{\mathscr{I}} .
$$

(v) We denote by $(\mathfrak{H}, \pi, \Omega)$ the GNS triple (carrier space, representation, cyclical vector) corresponding to the state $\Psi$ on $\hat{\mathscr{B}}$.

Note. Although $\Psi$ may depend on the choice of $\mathscr{M}^{\prime}$, this choice will be irrelevant to our formulation of the properties of $\phi$ (e.g. in Proposition 4.1).

Definition 4.3. For $M \in \mathscr{M}$, let $\lambda_{M}, \Sigma_{M}$ denote the second largest eigenvalue and the spectral set, respectively, of $\hat{v}_{M}$; and let $\Sigma\left(\mathscr{M}^{\prime}\right)$ $=\left\{\Sigma_{M} \mid M \in \mathscr{M}^{\prime}\right\}$. Then:

(i) We say that the principal eigenvalue of $\hat{v}_{M}$ is $\mathscr{M}_{0}$-asymptotically degenerate (resp. non-degenerate) ${ }^{5}$ if $\mathscr{M}$ contains a subcover, $\mathscr{M}_{0}$, of $T$ such that $\mathscr{M}_{0}-\lim _{M \rightarrow \infty} \lambda_{M}=1$ (resp. $\neq 1$ ). Note that there might exist subcovers $\mathscr{M}_{0}^{\prime}, \mathscr{M}_{0}^{\prime \prime}$ of $T$ such that the principal eigenvalue is asymptotically degenerate with respect to $\mathscr{M}_{0}^{\prime}$ and non-degenerate with respect to $\mathscr{M}_{0}^{\prime \prime}$.

${ }^{4}$ That equation (4.9) uniquely defines $\Psi$ follows from the fact that $\hat{\mathscr{B}}_{\mathscr{I}}$ is dense in $\hat{\mathscr{B}}$.

5 The significance of asymptotic degeneracy has been emphasised by Kac (cf. Ref. [6]). 
(ii) We say that $\Sigma\left(\mathscr{M}^{\prime}\right)$ possesses the property $Q$ if, for sufficiently large $M \in \mathscr{M}^{\prime}, \Sigma_{M}$ is given by $\Sigma_{M}^{(1)} \cup \Sigma_{M}^{(2)}$, such that:

(a) $\Sigma_{M}^{(1)} \subset[0, \Delta]$, where $\Delta(<1)$ is independent of $M$; and

(b) $\Sigma_{M}^{(2)} \subset\left[d_{M}, 1\right]$, where $\mathscr{M}^{\prime}-\lim _{M \rightarrow \infty} d_{M}=1$.

We define $\Delta_{0}$ to be the infimum of the values of $\Delta$ for which $\Sigma_{M}$ can be expressed as such a union.

(iii) We say that $\Sigma\left(\mathscr{M}^{\prime}\right)$ possesses the property $\bar{Q}$ if it satisfies $Q$ and if, in addition, $\exists \hat{B}_{1}, \ldots, \hat{B}_{n+1} \in \hat{\mathscr{B}}_{g}^{(0)}$ and $l_{1}, \ldots, l_{n} \in Z_{+}, n<\infty$ and, for any $\lambda<\Delta_{0},\left\|\left(\hat{E}_{M}\left(\Delta_{0}\right)-\hat{E}_{M}(\lambda)\right) \hat{B}_{1} \hat{v}_{M}^{l_{1}} \hat{B}_{2} \hat{v}_{M}^{l_{2}} \ldots \hat{v}_{M}^{l_{n}} \hat{B}_{n} \hat{\Omega}_{M}\right\|$ does not converge to zero as $M\left(\in \mathscr{M}^{\prime}\right) \rightarrow \infty$.

Note. Definition 4.3 will not be involved until Section 5. For the moment we note that it follows ${ }^{6}$ from the Schultz-Mattis-Lieb (SML) [7] formulation of $\mathscr{J}_{2}$ that, in case (I), $\Sigma(\mathscr{M})$ possesses the property $\bar{Q}$ except at the transition temperature; and that the principal eigenvalue of $\hat{v}_{M}$ is $\mathscr{M}$-asymptotically degenerate (resp. non-degenerate) below (resp. above) that temperature.

Lemma 4.1. (i) There exists a unique linear map $w: \hat{\mathscr{B}}_{\mathscr{I}} \rightarrow \hat{\mathscr{B}}_{\mathscr{I}}$ such that, given $\hat{B} \in \hat{\mathscr{B}}_{\mathscr{I}}, \exists M_{0}(B) \in \mathscr{M}$.

$$
\begin{aligned}
w_{M} \hat{B} & =w \hat{B}, \quad \forall M \supset M_{0}(B) . \\
\Psi(w \hat{B}) & =\Psi(\hat{B}), \quad \forall \hat{B} \in \hat{\mathscr{B}}_{\mathscr{I}} .
\end{aligned}
$$

Proof. (i) Let $\hat{B} \in \hat{\mathscr{B}}_{\mathscr{I}}$, i.e. $\hat{B} \in \hat{\mathscr{B}}_{M^{\prime}}$ for some $M^{\prime} \in \mathscr{M}$. Then in view of property (P), Definition 2.5 and Eq. (4.3), $\hat{f}_{M}$ may be expressed in the forms

$$
\begin{aligned}
\hat{f}_{M} & =\hat{f}_{v\left(M^{\prime}\right)}^{(1)}+\hat{f}_{M \backslash M^{\prime}}^{(2)} \\
& =\hat{f}_{v\left(v\left(M^{\prime}\right)\right)}^{(3)}+\hat{f}_{M \backslash v\left(M^{\prime}\right)}^{(4)}
\end{aligned}
$$

where

$$
\begin{aligned}
& \hat{f}_{v\left(M^{\prime}\right)}^{(1)} \in \hat{\mathscr{B}}_{v\left(M^{\prime}\right)}^{(0)}, \quad \hat{f}_{M \backslash M^{\prime}}^{(2)} \in \hat{\mathscr{B}}_{M \backslash M^{\prime}}^{(0)}, \\
& \hat{f}_{v\left(v\left(M^{\prime}\right)\right)}^{(3)} \in \hat{\mathscr{B}}_{v\left(v\left(M^{\prime}\right)\right)}^{(0)} \text { and } \hat{f}_{M \backslash v\left(M^{\prime}\right)}^{(4)} \in \hat{\mathscr{B}}_{M \backslash v\left(M^{\prime}\right)}^{(0)} .
\end{aligned}
$$

Further, it follows from Eq. (2.12) that

$$
\hat{\sigma}_{M}^{(3)}=\hat{\sigma}_{M \backslash v\left(M^{\prime}\right)}^{(3)}+\hat{\sigma}_{v\left(M^{\prime}\right)}^{(3)} .
$$

Since the algebra $\hat{\mathscr{B}}^{(0)}$ is Abelian (being isomorphic with $\mathscr{B}$ ), and since $\hat{\mathscr{B}}$ possesses the local commutativity property (Eq. (2.9)), it follows from Eqs. (4.4), (4.7), (4.12), (4.12') and (4.13) that

$$
w_{M} \hat{B}=\hat{Q} \hat{B} \hat{Q}^{-1}, \forall M \supset v\left(v\left(M^{\prime}\right)\right)
$$

with $\hat{Q}=\exp \left(-\frac{1}{2} \beta \hat{f}_{v\left(v\left(M^{\prime}\right)\right)}^{(3)}\right) \exp \left(-J_{\beta}^{*} \hat{\sigma}_{v\left(M^{\prime}\right)}^{(3)}\right) \exp \left(-\frac{1}{2} \beta \hat{f}_{v\left(M^{\prime}\right)}^{(1)}\right)$.

${ }^{6}$ One can infer from $S M L$ that $\mathscr{J}_{2}$ possesses the property $\bar{Q}$, except at $\beta=\beta_{c}$, by considering the case where $n=1$ and $B_{1}\left(y_{T}\right)=y_{1}$. For, using the technique employed in the last part of our proof of Lemma 3.1, it follows easily that, in this case.

$$
\mathscr{M}-\lim _{M \rightarrow \infty}\left\|\left(E_{M}(\Delta)-E_{M}(\lambda)\right) \hat{B}_{1} \hat{\Omega}_{M}\right\| \neq 0 \text { for } \lambda<\Delta_{0}:
$$


Therefore, since $\hat{Q}$ is independent of $M$, the required result follows directly from Eq. (4.15).

(ii) It follows from (i), together with Definition 4.2 (ii), (iii) that, for $M \supset M_{0}(\hat{B}), \Psi_{M}(w \hat{B})=\Psi_{M}\left(w_{M} \hat{B}\right)=\Psi_{M}(\hat{B})$. Hence, by Eq. (4.9), $\Psi(w \hat{B})$ $=\Psi(\hat{B})$.

Q.E.D.

Lemma 4.2. (i) There exists a positive self-adjoint contraction, $\hat{v}$, of $\mathfrak{H}$, uniquely defined by the formula

$$
\text { (ii) } \quad \begin{aligned}
v \pi(\hat{B}) \Omega & =\pi(w \hat{B}) \Omega, \quad \forall \hat{B} \in \hat{\mathscr{B}}_{\mathscr{F}} . \\
\hat{v} \Omega & =\Omega .
\end{aligned}
$$

Proof. (i) Let $v_{1}: \pi\left(\hat{\mathscr{B}}_{\mathscr{A}}\right) \Omega \rightarrow \mathfrak{H}$ be defined by:

$$
v_{1} \pi(\hat{B}) \Omega=\pi(w \hat{B}) \Omega, \quad \forall \hat{B} \in \hat{\mathscr{B}}_{\mathscr{I}} .
$$

Hence, by Definition 4.2 (i) -(v) and Lemma 4.1 (i), if $\hat{B} \in \hat{\mathscr{B}}_{\mathscr{I}}$, then $\left(\pi(\hat{B}) \Omega, v_{1} \pi(\hat{B}) \Omega\right)=\mathscr{M}^{\prime}-\lim _{M \rightarrow \infty} \Psi_{M}\left(\hat{B}^{*} w_{M} \hat{B}\right)=\mathscr{M}^{\prime}-\lim _{M \rightarrow \infty}\left(\hat{B} \hat{\Omega}_{M}, \hat{v}_{M} \hat{B} \hat{\Omega}_{M}\right)$.

Since $\hat{v}_{M}$ is a positive self-adjoint contraction of $\mathscr{H}_{M}$, it follows that the right-hand side of this equation is real, non-negative and

$$
\leqq \mathscr{M}^{\prime}-\lim _{M \rightarrow \infty}\left(\hat{B} \hat{\Omega}_{M}, \hat{B} \hat{\Omega}_{M}\right)=\mathscr{M}^{\prime}-\lim _{M \rightarrow \infty} \Psi_{M}\left(\hat{B}^{*} \hat{B}\right)=\Psi\left(\hat{B}^{*} \hat{B}\right)=\|\pi(\hat{B}) \Omega\|^{2} .
$$

Hence $\left(\pi(\hat{B}) \Omega, \quad v_{1} \pi(\hat{B}) \Omega\right) \in\left[0,\|\pi(\hat{B}) \Omega\|^{2}\right], \quad \forall \hat{B} \in \hat{\mathscr{B}}_{\mathscr{g}}$. Therefore, since $\pi\left(\hat{\mathscr{B}}_{\mathscr{g}}\right) \Omega$ is dense in $\mathfrak{H}, v_{1}$ admits a unique extension to a bounded, selfadjoint contraction of $\mathfrak{h}$. This contraction is the required operator $v$. (ii) follows on putting $B=I$ in Eq. (4.16), and noting that Definition 4.2 (ii) and Lemma 4.1 (i) imply that $w \hat{I}=\hat{I}$.

Q.E.D.

Definition 4.4. (i) For each $M \in \mathscr{M}$, we define the map $\hat{\theta}_{M}$ of $\bigcup_{M \in \mathscr{P}_{f}(Z)} \tilde{\mathscr{A}}_{M N}$ into $\mathscr{L}\left(\mathscr{H}_{M}\right)$ as follows. Let $A=\prod_{n \in N_{0}} B^{(n)} \in \tilde{\mathscr{A}}_{M N}$ (in the notation of Definition 2.2), with $N_{0}=\left\{n_{1}, n_{2}, \ldots, n_{k}\right\} \subset N \in \mathscr{P}_{f}(Z), n_{1}<n_{2} \cdots<n_{k}$, and the $B^{(n)}$ 's $\in \mathscr{B}_{M}$. Then, denoting $\delta\left(B^{(n)}\right)$ by $\hat{B}^{(n)}$,

$$
\hat{\theta}_{M}(A)=\hat{B}^{\left(n_{1}\right)} \hat{v}_{M}^{n_{2}-n_{1}} \hat{B}^{\left(n_{2}\right)} \hat{v}_{M}^{n_{3}-n_{2}} \hat{B}^{\left(n_{3}\right)} \ldots \hat{v}_{M}^{n_{k}-n_{k-1}} \hat{B}^{\left(n_{k}\right)} .
$$

(ii) We define the map $\theta$ of $\tilde{\mathscr{A}}$ into $\mathscr{L}(\mathfrak{H})$ as follows. Let $A=\prod_{n \in N_{0}} B^{(n)}$ $\in \tilde{\mathscr{A}}_{M N}$ as above. Then

$$
\theta(A)=\pi\left(\hat{B}^{\left(n_{1}\right)}\right) v^{n_{2}-n_{1}} \pi\left(\hat{B}^{\left(n_{2}\right)}\right) v^{n_{3}-n_{2}} \pi\left(\hat{B}^{\left(n_{3}\right)}\right) \ldots v^{n_{k}-n_{k-1}} \pi\left(\hat{B}^{\left(n_{k}\right)}\right) .
$$

Lemma 4.3. $\phi(A)=(\Omega, \theta(A) \Omega), \forall A \in \tilde{\mathscr{A}}$. 
Proof. Let $A=\prod_{n \in N_{0}} B^{(n)}$, as in Definition 2.2 with the $B^{(n)}$ s $\in \in \mathscr{B}_{M_{0}}$ and $M_{0} \in \mathscr{P}_{f}(T)$. Then it follows from Eq. (2.5)-(2.7), (3.1), (3.2), (3.7), (3.8), and (4.1) that, for $M_{0} \subset M \in \mathscr{M}$ and $N_{0} \subset N=[a, b] \in \mathscr{N} \equiv \mathscr{P}_{f}(Z)$,

$$
\begin{aligned}
& E_{M N}(A)=\sum_{y M \in Y_{M}^{N}}\left[\prod_{n=a}^{n_{1}-1} V_{M}\left(y_{M}^{(n)}, y_{M}^{(n+1)}\right)\right] B_{M}^{\left(n_{1}\right)}\left(y_{M}^{\left(n_{1}\right)}\right)\left[\prod_{n=n_{1}}^{n_{2}-1} V_{M}\left(y_{M}^{(n)}, y_{M}^{(n+1)}\right)\right] \\
& \times B_{M}^{\left(n_{2}\right)}\left(y_{M}^{\left(n_{2}\right)}\right) \times \cdots\left[\prod_{n=n_{k-1}}^{n_{k}-1} V_{M}\left(y_{M}^{(n)}, y_{M}^{(n+1)}\right)\right] B_{M}^{\left(n_{k}\right)}\left(y_{M}^{\left(n_{k}\right)}\right)\left[\prod_{n=n_{k}}^{b} V_{M}\left(y_{M}^{(n)}, y_{M}^{(n+1)}\right)\right]
\end{aligned}
$$

where $B_{M}^{(n)}=q_{M} B^{(n)}$, and $(b+1)$ is identified with $a$ in the last product. Thus, by Definitions 2.5, 2.6 and Eq. (4.2),

$$
\begin{aligned}
E_{M N}(A) & =\operatorname{Tr}_{\mathscr{H}_{M}}\left[\hat{B}^{\left(n_{1}\right)} \hat{V}_{M}^{n_{2}-n_{1}} \hat{B}^{\left(n_{2}\right)} \hat{V}_{M}^{n_{3}-n_{2}} \ldots \hat{B}^{\left(n_{k}\right)} \hat{V}_{M}^{b-a-n_{k}+n_{1}+1}\right] \\
& =\Lambda_{M}^{b-a+1} \operatorname{Tr}_{\mathscr{H}_{M}}\left[\hat{v}_{M}^{b-a+1} \hat{\theta}_{M}(A)\right] ;
\end{aligned}
$$

and therefore, by Eq. (3.6),

$$
\mathcal{N}-\lim _{N \rightarrow \infty} \phi_{M N}(A)=\lim _{n \rightarrow \infty} \operatorname{Tr}_{\mathscr{H}_{M}}\left[\hat{v}_{M}^{n} \hat{\theta}_{M}(A)\right] / \operatorname{Tr}_{\mathscr{H}_{M}}\left[\hat{v}_{M}^{n}\right] .
$$

Further, by Definition 4.2, $\hat{v}_{M}^{n}$ tends normwise to the projection operator for $\hat{\Omega}_{M}$, as $n \rightarrow \infty$. Hence, it follows from Eq. (4.21) and the finite-dimensionality of $\mathscr{H}_{M}$ that

$$
\mathscr{N}-\lim _{N \rightarrow \infty} \phi_{M N}(A)=\left(\hat{\Omega}_{M}, \hat{\theta}_{M}(A) \hat{\Omega}_{M}\right) .
$$

By Definitions 4.2 (ii) and 4.4 (i),

$$
\hat{\theta}_{M}(A) \hat{\Omega}_{M}=\hat{B}^{\left(n_{1}\right)}\left(w_{M}^{n_{2}-n_{1}} \hat{B}^{\left(n_{2}\right)}\right) \ldots\left(w_{M}^{n_{k}-n_{1}} \hat{B}^{\left(n_{k}\right)}\right) \hat{\Omega}_{M} .
$$

Hence, it follows from Lemma 4.1 (i) that, for $M$ sufficiently large,

$$
\hat{\theta}_{M}(A) \hat{\Omega}_{M}=\hat{B}^{\left(n_{1}\right)}\left(w^{n_{2}-n_{1}} \hat{B}^{\left(n_{2}\right)}\right) \ldots\left(w^{n_{k}-n_{1}} \hat{B}^{\left(n_{k}\right)}\right) \hat{\Omega}_{M} .
$$

Thus, defining

$$
\kappa_{r}(A)=\hat{B}^{\left(n_{r}\right)}\left(w^{n_{r+1}-n_{r}} \hat{B}^{\left(n_{r+1}\right)}\right) \ldots\left(w^{n_{k}-n_{r}} \hat{B}^{\left(n_{k}\right)}\right), \text { for } \quad r=1, \ldots, k,
$$

we see that $\left(\hat{\Omega}_{M}, \hat{\theta}_{M}(A) \hat{\Omega}_{M}\right)=\left(\hat{\Omega}_{M}, \kappa_{1}(A) \hat{\Omega}_{M}\right)$; and therefore, by Eq. (4.8) and (4.22),

$$
\mathscr{N}-\lim _{N \rightarrow \infty} \phi_{M N}(A)=\Psi_{M}\left(\kappa_{1}(A)\right) .
$$

It now follows from Eqs. (3.9), (4.24), together with Definitions 4.2 (iii)-(v), that

$$
\phi(A)=\mathscr{M}^{\prime}-\lim _{M \rightarrow \infty} \mathcal{N}-\lim _{N \rightarrow \infty} \phi_{M N}(A)=\left(\Omega, \pi\left(\kappa_{1}(A)\right) \Omega\right) .
$$


Moreover, by Definition 4.2 (ii), Lemma 4.1 (i) and Eq. (4.23),

$$
\pi\left(\kappa_{r}(A)\right) \Omega=\pi\left(\hat{B}^{\left(n_{r}\right)}\right) v^{n_{r+1}-n_{r}} \pi\left(\kappa_{r+1}(A)\right) \Omega, \text { for } \quad r=1, \ldots, k-1 .
$$

Thus, $\pi\left(\kappa_{1}(A)\right) \Omega=\pi\left(\hat{B}^{\left(n_{1}\right)}\right) v^{n_{2}-n_{1}} \pi\left(\hat{B}^{\left(n_{2}\right)}\right) \ldots v^{n_{k}-n_{k}-1} \pi\left(\hat{B}^{\left(n_{k}\right)}\right) \Omega$, i.e., by Definition 4.2 (ii),

$$
\pi\left(\kappa_{1}(A)\right) \Omega=\theta(A) \Omega .
$$

Eq. (4.20) follows directly from Eqs. (4.25), (4.26).

Q.E.D.

Definition 4.5. (i) We define $\mathfrak{H}_{0}$ to be the subspace of $\mathfrak{H}$ generated by linear combinations of vectors $v^{l_{1}} \pi\left(\hat{B}_{1}\right) v^{l_{2}} \pi\left(\hat{B}_{2}\right) \ldots v^{l_{r}} \pi\left(\hat{B}_{r}\right) \Omega$, with $r, l_{1}, \ldots, l_{r} \in Z_{+}$and $\hat{B}_{1}, \ldots, \hat{B}_{r} \in \hat{\mathscr{B}}^{(0)}$. Thus, $\mathfrak{H}_{0}$ is stable under both $v$ and $\pi\left(\hat{\mathscr{B}}^{(0)}\right)$.

(ii) We denote the restriction of $v$ to $\mathfrak{S}_{0}$ by $v_{0}$.

(iii) We define $E_{0}$ to be the projection operator for the subspace of $\mathfrak{S}_{0}$ spanned by vectors that are invariant under $v_{0}$. (Thus, by Lemma 4.2 (ii), $\Omega \in E_{0} \mathfrak{H}_{0}$.)

(iv) We define $v_{0}^{\prime}=v_{0}-E_{0}$.

(v) We define maps $\alpha, \alpha^{*}$ of $\tilde{\mathscr{A}}$ into $\mathfrak{H}_{0}$ by the formulae

$$
\alpha(A)=\theta(A) \Omega ; \alpha^{*}(A)=\theta(A)^{*} \Omega ; \forall A \in \tilde{\mathscr{A}} .
$$

Lemma 4.4. $\alpha(\tilde{\mathscr{A}})\left(\equiv \alpha^{*}(\tilde{\mathscr{A}})\right)$ is a basis set in $\mathfrak{H}_{0}$.

Proof. It follows from Definitions 4.4 (ii) and 4.5 (v) that $\alpha(\tilde{\mathscr{A}})=\alpha^{*}(\tilde{\mathscr{A}})$ $=\left\{\pi(\hat{B}) v^{l_{1}} \pi\left(\hat{B}_{1}\right) v^{l_{2}} \ldots v^{l_{r}} \pi\left(\hat{B}_{r}\right) \Omega \mid r, l_{1}, \ldots, l_{r} \in Z_{+} ; \hat{B}, \hat{B}_{1}, \ldots, \hat{B}_{r} \in \hat{\mathscr{B}}_{\mathscr{g}}^{(0)} \backslash \hat{I}\right\}$. Hence, since $\hat{\mathscr{B}}_{\mathscr{G}}^{(0)} \backslash \hat{I}$ is norm-dense in $\hat{\mathscr{B}}^{(0)}$, it follows from Definition 4.5 (i) that $\alpha(\tilde{\mathscr{A}})\left(\equiv \alpha^{*}(\tilde{\mathscr{A}})\right)$ forms a basis set in $\mathfrak{S}_{0}$.

Proposition 4.1. (i) $\phi(A)=(\Omega, \alpha(A))=\left(\alpha^{*}(A), \Omega\right), \forall A \in \tilde{\mathscr{A}}$.

(ii) Given $A^{\prime}, A \in \tilde{\mathscr{A}}, \exists n_{0} \equiv n_{0}\left(A^{\prime}, A\right) \in Z$ such that for $n_{0}<n(\in Z)$,

$$
F\left(A^{\prime}, A ; n\right)=\left(\alpha^{*}\left(A^{\prime}\right), v_{0}^{n-n_{0}} \alpha(A)\right) \text {. }
$$

(iii) $F_{\eta}\left(A^{\prime}, A\right)=\left(\alpha^{*}\left(A^{\prime}\right), E_{0} \alpha(A)\right) ; \forall A^{\prime}, A \in \tilde{\mathscr{A}}$.

(iv) $F^{\prime}\left(A^{\prime}, A ; n\right)=\left(\alpha^{*}\left(A^{\prime}\right),\left(v_{0}^{\prime}\right)^{n-n_{0}} \alpha(A)\right) ; \forall A^{\prime}, A \in \tilde{\mathscr{A}}, n>n_{0}$.

Proof. (i) follows from Lemma 4.3 and Definition 4.5 (v).

and

(ii) Let $A, A^{\prime} \in \tilde{\mathscr{A}}$, where $A=\prod_{n \in N_{0}} B^{(n)}$, as specified in Definition 4.4, $A^{\prime}=\prod_{n \in N_{0}^{\prime}} B^{\prime(n)}$, with $N_{0}^{\prime}=\left\{n_{1}^{\prime}, \ldots, n_{l}^{\prime}\right\} \in \mathscr{P}_{f}(Z) \quad$ and $\quad n_{1}^{\prime}<n_{2}^{\prime}<\cdots<n_{l}^{\prime}$.

Denoting $n_{l}^{\prime}-n_{1}$ by $n_{0}\left(\equiv n_{0}\left(A^{\prime}, A\right)\right)$, it follows from Definitions 2.3 (i) and 4.4 (ii) that, for $n>n_{0}, \theta\left(A^{\prime} \tau_{n} A\right)=\theta\left(A^{\prime}\right) v^{n-n_{0}} \theta(A)$. Hence, it follows from Lemma 4.3 and Definition 3.3 (i) that

$$
F\left(A^{\prime}, A ; n\right)=\left(\Omega, \theta\left(A^{\prime}\right) v^{n-n_{0}} \theta(A) \Omega\right) .
$$


Consequently, by Definition 4.5 (ii), (v),

$$
F\left(A^{\prime}, A ; n\right)=\left(\alpha^{*}\left(A^{\prime}\right), v_{0}^{n-n_{0}} \alpha(A)\right),
$$

as required.

(iii) It follows from (ii) that, for $m\left(\in Z_{+}\right)>n_{0}$,

$$
\left.\begin{array}{rl}
\sum_{n=1}^{m}\left[F\left(A^{\prime}, A ; n\right)-\left(\alpha^{*}\left(A^{\prime}\right), v_{0}^{n-n_{0}} \alpha(A)\right)\right] & =\left(\sum_{n=1}^{n_{0}}-\sum_{n=m+1}^{m+n_{0}}\right) F\left(A^{\prime}, A ; n\right), \\
& =0, \quad \text { for } \quad n_{0}>0, \\
& =\left(\sum_{n=m+1}^{m-n_{0}}-\sum_{n=1}^{-n_{0}}\right)\left(\alpha^{*}\left(A^{\prime}\right), v_{0}^{n} \alpha(A)\right), \\
& \text { for } \quad n_{0}<0 .
\end{array}\right\}
$$

Further, it follows from Definitions 3.3 (i), 4.4 (ii) and 4.5 (v) that, since $v_{0}$ is a contraction, $\left|F\left(A^{\prime}, A ; n\right)\right|$ and $\left|\left(\alpha^{*}\left(A^{\prime}\right), v_{0}^{n} \alpha(A)\right)\right|$ are both $\leqq\|A\|\left\|A^{\prime}\right\|$, $\forall n \in Z_{+}$. Hence, by Eq. (4.33),

$$
\left|\sum_{n=1}^{m} F\left(A^{\prime}, A ; n\right)-\sum_{n=1}^{m}\left(\alpha^{*}\left(A^{\prime}\right), v_{0}^{n} \alpha(A)\right)\right| \leqq\left|n_{0}\right|\|A\|\left\|A^{\prime}\right\|,
$$

irespective of whether $n_{0}>$ or $=$ or $<0$.

Next we note that, since $v_{0}$ is a contraction of $\mathfrak{H}_{0}$, it follows from Definition 4.5 (iii) that (cf. Ref. [8], p. 409)

$$
\left(s, \mathfrak{H}_{0}\right)-\lim _{m \rightarrow \infty} \frac{1}{m} \sum_{n=1}^{m} v_{0}^{n}=E_{0} ;
$$

and therefore, by the inequality (4.34),

$$
\lim _{m \rightarrow \infty} \frac{1}{m} \sum_{n=1}^{m} F\left(A^{\prime}, A ; n\right)=\left(\alpha^{*}\left(A^{\prime}\right), E_{0} \alpha(A)\right) .
$$

The required result follows from this last equation, together with Definition 3.3 (ii).

(iv) follows from (ii) and (iii), together with Definitions 3.3 (iii) and 4.5 (iv).

Q.E.D.

Corollary. $\left(\ln \left\|v_{0}^{\prime}\right\|^{-1}\right)^{-1}=\sup _{A^{\prime}, A \in \mathscr{A}}\left\{r \mid r \in R_{+} ; \lim _{n \rightarrow \infty} F^{\prime}\left(A^{\prime}, A ; n\right) e^{n / r^{\prime}}\right.$

$$
\left.=0 \text { for } r<r^{\prime} \in R_{+}\right\} \text {. }
$$

Proof. Since $\alpha(\tilde{\mathscr{A}})\left(\equiv \alpha^{*}(\tilde{\mathscr{A}})\right)$ is a basis set in $\mathfrak{H}_{0}$, and since $v_{0}^{\prime}$ is a positive self-adjoint operator in $\mathfrak{S}_{0}$, it follows from Proposition 4.1 (iv) 
that

$$
\left(\ln \left\|v_{0}^{\prime}\right\|^{-1}\right)^{-1}=\sup _{A^{\prime}, A \in \tilde{A}}\left\{r \mid r \in R_{+} ; \lim _{n \rightarrow \infty} F^{\prime}\left(A^{\prime}, A ; n\right) e^{n / r^{\prime}}=0 \text { for } r<r^{\prime} \in R_{+}\right\} .
$$

Since $\tilde{\mathscr{A}}$ forms a basis set in $\mathscr{A}$, it follows from Definition 3.3 (iii) that this last equation may be extended so as to yield the required result. Q.E.D.

In view of this Corollary and of the significance of $F^{\prime}$ (cf. Note at the end of Section 3), we introduce the following definition.

Definition 4.6. (i) We define $l=\left(\ln \left\|v_{0}^{\prime}\right\|^{-1}\right)^{-1}$ to be the correlation length for the state $\phi$.

(ii) For $A^{\prime}, A \in \mathscr{A}$, we define the correlation length

$$
l_{A^{\prime} A}=\sup \left\{r \mid r \in R_{+} ; \lim _{n \rightarrow \infty} F^{\prime}\left(A^{\prime}, A ; n\right) e^{n / r^{\prime}}=0 \quad \text { for } \quad r<r^{\prime} \in R_{+}\right\} \text {. }
$$

Thus, by the Corollary to Proposition 4.1, $l=\sup _{A^{\prime}, A \in \mathscr{A}} l_{A^{\prime} A^{\prime}}$

Proposition 4.2. (i) $\phi$ is G-ergodic if and only if the projector $E_{0}$ is one-dimensional, i.e. if $E_{0}$ is the projection operator for $\Omega$.

(ii) Let $\mathfrak{H}_{0}^{(-)}$be the subspace of $\mathfrak{H}_{0}$ generated by $\alpha\left(\mathscr{A}^{(-)}\right)$. Then $F_{\eta}$ vanishes on $\mathscr{A}^{(-)} \times \mathscr{A}^{(-)}$if and only if the spaces $E_{0} \mathfrak{H}_{0}, \mathfrak{H}_{0}^{(-)}$are not mutually orthogonal; in which case, if $\phi \in \mathfrak{6}_{\varrho}$, then (by Lemma 4.2), it undergoes a @-symmetry breakdown associated with its G-ergodic decomposition.

Proof. (i) It follows from Definition 3.3 (ii) that $\phi$ is $G$-ergodic (i.e. weakly clustering) if and only if $F_{\eta}\left(A^{\prime}, A\right)=\phi\left(A^{\prime}\right) \phi(A), \forall A^{\prime}, A \in \mathscr{A}$. Since $\tilde{A}$ is a basis for $\mathscr{A}$, this ergodicity condition is equivalent to:

$$
F_{\eta}\left(A^{\prime}, A\right)=\phi\left(A^{\prime}\right) \phi(A), \forall A^{\prime}, A \in \tilde{\mathscr{A}} ;
$$

i.e. by Proposition 4.1 (i), (iii), it is equivalent to the condition that

$$
\left(\alpha^{*}\left(A^{\prime}\right),\left(E_{0}-E(\Omega)\right) \alpha(A)\right)=0 ; \forall A^{\prime}, A \in \tilde{\mathscr{A}},
$$

where $E(\Omega)$ is the projection operator for $\Omega$. Thus, since $\alpha(\tilde{\mathscr{A}}) \equiv \alpha^{*}(\tilde{\mathscr{A}})$ is a basis set in $\mathfrak{H}_{0}$, it follows that $\phi$ is $G$-ergodic if and only if $E_{0}=E(\Omega)$, as required.

(ii) Since $\tilde{\mathscr{A}}^{(-)}$is a basis set in $\mathscr{A}^{(-)}$, it follows from Definition 3.3 (ii) that $F_{\eta}$ vanishes on $\mathscr{A}^{(-)} \times \mathscr{A}^{(-)}$if and only if vanishes on $\tilde{\mathscr{A}}^{(-)} \times \tilde{\mathscr{A}}^{(-)}$. By Definition $4.5(\mathrm{v}), \alpha\left(\tilde{\mathscr{A}}^{(-)}\right)=\alpha^{*}\left(\tilde{\mathscr{A}}^{(-)}\right)$; and by Proposition 4.1 (iii), $F_{\eta}\left(A^{\prime}, A\right)=\left(\alpha^{*}\left(A^{\prime}\right), E_{0} \alpha(A)\right), \forall A^{\prime}, A \in \tilde{\mathscr{A}}^{(-)}$. Hence, $F_{\eta}$ vanishes on $\tilde{\mathscr{A}}^{(-)}$ $\times \tilde{A}^{(-)}$, and thus on $\mathscr{A}^{(-)} \times \mathscr{A}^{(-)}$, if and only if $E_{0} \alpha\left(\tilde{A}^{(-)}\right)=0$, i.e. if the spaces $E_{0} \mathfrak{H}_{0}$ and $\mathfrak{S}_{0}^{(-)}$are mutually orthogonal.

Q.E.D.

Proposition 4.3. Let $\Sigma\left(\mathscr{M}^{\prime}\right)$ possess the property $Q$ (specified in Definition 4.3). Then:

(i) The correlation length $l \geqq\left(\ln \left(\Delta_{0}^{-1}\right)\right)^{-1}$. 
(ii) If $\Sigma\left(\mathscr{M}^{\prime}\right)$ possesses the property $\bar{Q}$, then $l=\left(\ln \left(\Delta_{0}^{-1}\right)\right)^{-1}$.

(iii) If the principal eigenvalue of $\hat{v}_{M}$ is $\mathscr{M}_{0}$-asymptotically nondegenerate for some $\mathscr{M}_{0} \subset \mathscr{M}^{\prime}$, then $\phi$ is G-ergodic.

Proof. (i) By Eqs. (3.9) and (4.22),

$$
F\left(A^{\prime}, A ; n\right)=\mathscr{M}^{\prime}-\lim _{M \rightarrow \infty} F_{M}\left(A^{\prime}, A ; n\right), \forall A^{\prime}, A \in \tilde{\mathscr{A}}, n \in Z,
$$

where $F_{M}\left(A^{\prime}, A ; n\right)=\left(\hat{\Omega}_{M}, \hat{\theta}_{M}\left(A^{\prime} \tau_{n} A\right) \hat{\Omega}_{M}\right), \forall A^{\prime}, A \in \bigcup_{N \in \mathscr{P}_{f}(Z)} \tilde{\mathscr{A}}_{M N}, n \in Z$.

Defining $n_{0}$ as in Proposition 4.1 (ii), it follows from Definitions 2.3 (i), 4.4 (i) that $\hat{\theta}_{M}\left(A^{\prime} \tau_{n} A\right)=\hat{\theta}_{M}\left(A^{\prime}\right) \hat{v}_{M}^{n-n_{0}} \hat{\theta}_{M}(A), \forall n>n_{0}$. Hence, by Eq. (4.36),

$$
F_{M}\left(A^{\prime}, A ; n\right)=\left(\hat{\Omega}_{M}, \hat{\theta}_{M}\left(A^{\prime}\right) \hat{v}_{M}^{n-n_{0}} \hat{\theta}_{M}(A) \hat{\Omega}_{M}\right), \text { for } n>n_{0} .
$$

Assuming now that $\Sigma\left(\mathscr{M}^{\prime}\right)$ possesses the property $Q$, it follows from Definition 4.3 (ii) that, for sufficiently large $M\left(\in \mathscr{M}^{\prime}\right)$, we may express $\hat{v}_{M}$ in the form

$$
\hat{v}_{M}=\hat{v}_{M}^{(1)}+\hat{v}_{M}^{(2)}
$$

where $\quad \hat{v}_{M}^{(1)}=\int_{-0}^{\Delta} \lambda d \hat{E}_{M}(\lambda) ; \quad$ and $\quad v_{M}^{(2)}=\int_{d_{M}-0}^{1} \lambda d \hat{E}_{M}(\lambda)$.

Let $\hat{E}_{M}^{(2)}$ be the projector defined by:

$$
\hat{E}_{M}^{(2)}=\hat{E}_{M}(1)-\hat{E}_{M}\left(d_{M}-0\right) .
$$

Then since, by Definition 4.3 (ii), $\mathscr{M}^{\prime}-\lim _{M \rightarrow \infty} d_{M}=1$, it follows from Eqs. (4.38)-(4.40) that

$$
\mathscr{M}^{\prime}-\lim _{M \rightarrow \infty}\left\|\hat{v}_{M}^{n-n_{0}}-\left(\hat{v}_{M}^{(1)}\right)^{n-n_{0}}-\hat{E}_{M}^{(2)}\right\|=0, \quad \forall n>n_{0} .
$$

Consequently, by Eqs. (4.35)-(4.37),

$$
\begin{aligned}
& F\left(A^{\prime}, A ; n\right)=\mathscr{M}^{\prime}-\lim _{M \rightarrow \infty}\left[F_{M}^{(1)}\left(A^{\prime}, A ; n\right)+F_{M}^{(2)}\left(A^{\prime}, A\right)\right] ; \forall A^{\prime}, A \in \tilde{\mathscr{A}} ; n>n_{0}, \\
& \text { where } \\
& \text { and }
\end{aligned}
$$

Further, it follows from Definition 4.4 (i) and Eqs. (4.39), (4.42) and (4.43) that

$$
\left|F_{M}^{(1)}\left(A^{\prime}, A ; n\right)\right| \leqq\left\|A^{\prime}\right\|\|A\| \Delta^{n-n_{0}}, \text { for } n>n_{0},
$$

and

$$
\left|F_{M}^{(2)}\left(A^{\prime}, A\right)\right| \leqq\left\|A^{\prime}\right\|\|A\| \text {. }
$$


In view of this latter equation, it follows from the BolzanoWeierstrass theorem that $\mathscr{M}^{\prime}$ contains a subcover, $\mathscr{M}^{\prime \prime}$, of $T$ such that $\mathscr{M}^{\prime \prime}-\lim _{M \rightarrow \infty} F_{M}^{(2)}\left(A^{\prime}, A\right)$ exists. Hence, defining

$$
F^{(2)}\left(A^{\prime}, A\right)=\mathscr{M}^{\prime \prime}-\lim _{M \rightarrow \infty} F_{M}^{(2)}\left(A^{\prime}, A\right),
$$

it follows from Eq. (4.41) that

$F\left(A^{\prime}, A ; n\right)=F^{(2)}\left(A^{\prime}, A\right)+\mathscr{M}^{\prime \prime}-\lim _{M \rightarrow \infty} F_{M}^{(1)}\left(A^{\prime}, A ; n\right), \forall A^{\prime}, A \in \tilde{\mathscr{A}}, n>n_{0}$.

Since $\Delta<1$ (cf. Definition 4.3 (ii)), it follows from Definition 3.3 (ii) and Eqs. (4.44), (4.47) that

$$
F_{\eta}\left(A^{\prime}, A\right)=F^{(2)}\left(A^{\prime}, A\right), \forall A^{\prime}, A \in \tilde{\mathscr{A}}
$$

and thus, by Definition 3.3 (iii) and Eq. (4.47),

$$
F^{\prime}\left(A^{\prime}, A ; n\right)=\mathscr{M}^{\prime \prime}-\lim _{M \rightarrow \infty} F_{M}^{(1)}\left(A^{\prime}, A ; n\right), \quad \forall A^{\prime}, A \in \tilde{\mathscr{A}}, \quad n>n_{0} .
$$

Consequently, by Definition 4.6 (ii) and Eqs. (4.44), (4.49), $l_{A^{\prime} A} \leqq \Delta$ and thus (by Definition 4.3 (ii)) $\leqq \Delta_{0}, \forall A^{\prime}, A \in \mathscr{A}$.

Since $\tilde{\mathscr{A}}$ is a basis set in $\mathscr{A}$, this inequality may be extended to all $A^{\prime}, A \in \mathscr{A}$, and therefore $l \leqq\left(\ln \left(\Delta_{0}^{-1}\right)\right)^{-1}$.

(ii) It follows from Definitions 4.3 (ii), 4.4 (i), Eqs. (4.42), (4.49) and the Bolzano-Weierstrass theorem that, if $\Sigma\left(\mathscr{M}^{\prime}\right)$ possesses the property $\bar{Q}$, then $\exists A^{\prime}, A \in \tilde{\mathscr{A}}$ such that $l_{A^{\prime} A}=\left(\ln \left(\Delta_{0}^{-1}\right)\right)^{-1}$. Thus, in view of $(\mathrm{i})$, $l=\left(\ln \left(\Delta_{0}^{-1}\right)\right)^{-1}$.

(iii) Assume that the principle eigenvalue of $\hat{v}_{M}$ is $\mathscr{M}_{0}$-asymptotically degenerate, with $\mathscr{M}_{0} \subset \mathscr{M}^{\prime}$. Then it follows from Definition 4.3 (i) and Eq. (4.43) that

$$
F_{M}^{(2)}\left(A^{\prime}, A\right)=\left(\hat{\Omega}_{M}, \hat{\theta}_{M}\left(A^{\prime}\right) \hat{\Omega}_{M}\right)\left(\hat{\Omega}_{M}, \hat{\theta}_{M}(A) \hat{\Omega}_{M}\right), \quad \text { for } \quad M \in \mathscr{M}_{0},
$$

and hence, by Eqs. (3.9), (4.22),

$$
\mathscr{M}_{0}-\lim _{M \rightarrow \infty} F_{M}^{(2)}\left(A^{\prime}, A\right)=\phi\left(A^{\prime}\right) \phi(A) .
$$

Thus, the set $\mathscr{M}^{\prime \prime}$, which was introduced in the proof of (i), may be taken to be $\mathscr{M}_{0}$; and hence, by Eqs. (4.48), (4.50),

$$
F_{\eta}\left(A^{\prime}, A\right)=\phi\left(A^{\prime}\right) \phi(A), \forall A^{\prime}, A \in \tilde{\mathscr{A}} .
$$

Since $\tilde{\mathscr{A}}$ is a basis set in $\mathscr{A}$, this last equation implies that

$$
F_{\eta}\left(A^{\prime}, A\right)=\phi\left(A^{\prime}\right) \phi(A), \forall A^{\prime}, A \in \mathscr{A} ;
$$

and thus that $\phi$ is $G$-ergodic (weakly clustering).

Q.E.D. 


\section{Application to Phase Transitions}

The following properties of the Gibbs state have now been established:

(1) (cf. Ref. [1]). The specific free energy, $f$, is given by

$$
\mathscr{M}-\lim _{M \rightarrow \infty}(-\beta) N_{M}^{-1} \ln \Lambda_{M},
$$

where $\Lambda_{M}$ is the principal eigenvalue of $\hat{V}_{M}$ and $N_{M}$ is the number of sites in $M$.

(2) $\phi$ is $G$-ergodic if and only if the projector $E_{0}$ is one-dimensional (Proposition 4.2 (i)). Further, this condition is fulfilled if the principal eigenvalue of $\hat{v}_{M}$ is $\mathscr{M}_{0}$-asymptotically non-degenerate for some subcover $\mathscr{M}_{0}\left(\subset \mathscr{M}^{\prime}\right)$ of $T$ (Proposition 4.3 (iii)).

(3) If the spaces $E_{0} \mathfrak{H}_{0}, \mathfrak{H}_{0}^{(-)}$are not mutually orthogonal (i.e. if $F_{\eta}$ does not vanish on $\left.\mathscr{A}^{(-)} \times \mathscr{A}^{(-)}\right)$then, if $\phi \in \mathfrak{G}_{o}$, it undergoes a $\varrho$-symmetry breakdown associated with its $G$-ergodic decomposition (Proposition 4.2 (ii)).

(4) The correlation length $l$ is given by $\left(\ln \left\|v_{0}^{\prime}\right\|^{-1}\right)^{-1}$ (Definition 4.6 (i)). In cases where the system possesses property $\bar{Q}, l=\left(\ln \Delta_{0}^{-1}\right)^{-1}$ (Proposition 4.3 (ii)).

Let us now consider cases where $\phi \in \mathfrak{b}_{e}$ and the system possesses the following properties (a)-(d), which have already been established ${ }^{7}$ for the model $\mathscr{J}_{2}$ (our Case (I)):

(a) $f$ has a singularity at a unique inverse temperature $\beta_{c}$.

(b) The principal eigenvalue of $\hat{v}_{M}$ is $\mathscr{M}$-asymptotically nondegenerate for $\beta<\beta_{c}$.

(c) $F_{\eta}$ does not vanish on $\mathscr{A}^{(-)} \times \mathscr{A}^{(-)}$for $\beta>\beta_{c}$.

(d) $\Sigma(\mathscr{M})$ possesses the property $\bar{Q}$; and further $\Delta_{0}$ is a continuous function of $\beta$, which takes the value unity at $\beta=\beta_{c}$. It follows from (1)-(4) that the properties (a)-(d) ensure that the system exhibits a phase transition at $\beta=\beta_{c}$, characterised by:

(A) a thermodynamical singularity;

(B) a change from $G$-ergodicity of the $\left(\varrho\right.$-invariant) state $\phi\left(\beta<\beta_{c}\right)$ to a $\varrho$-symmetry breakdown of $\phi$ associated with its $G$-ergodic decomposition $\left(\beta>\beta_{c}\right)^{8}$;

(C) a divergence of $l$ as $\beta \rightarrow \beta_{c} \pm 0$.

Thus if $\phi \in \mathfrak{G}_{e}$ the properties (a)-(d) ensure that the system exhibits a phase transition characterised by (A), (B) and (C). It should be em-

${ }^{7}$ For the model $\mathscr{J}_{2}$, (a) is proved by Onsager [1]; (b) is proved by $S M L$; (c) follows from the $S M L$ treatment of the asymptotic properties of the correlation function governing a pair of spins; and (cf. our footnote (6)), (d) also follows from $S M L$.

${ }^{8}$ A related result for the model $\mathscr{J}_{2}$ has been obtained by Emch, Knops and Verboven [9], who show that, when $\beta>\beta_{c}$, the restriction of $\phi$ to a certain linear manifold of observables for one row decomposes into weakly-clustering states of equal and opposite non-zero polarisation. 
phasised that the concurrence of these three characteristics is rather remarkable, since they depend on apparently unrelated features of the model. For the free energy is governed by the principal eigenvalue of $\hat{V}_{M}$, the $G$-ergodic properties by the dimensionality of $E_{0} \mathfrak{H}_{0}$, and the length $l$ by the gap between the principal eigenvalue and the rest of the spectrum of $v_{0}$.

Finally, we note that, if $G$ is a subgroup of a larger amenable ${ }^{9}$ group $H$, then $G$-ergodicity implies $H$-ergodicity. On the other hand, the $H$-ergodic decomposition is not necessarily a refinement of the $G$-ergodic one; and therefore a $\varrho$-symmetry breakdown associated with the latter decomposition does not ensure the existence of such a breakdown associated with the former one. Consequently, further information is needed if one is to extend the above conclusions from $G$ to $H$. In the particular case of the model $\mathscr{J}_{2}$, with $H$ taken to correspond to the lattice group $Z^{2}$, such information is provided by SML's result that the asymptotic form of the space-correlation between a pair of spins is independent of the direction of their relative displacement. Hence, one easily concludes that, for $\beta>\beta_{c}$, the system undergoes a $\varrho$-symmetry breakdown associated with its $H$-ergodic decomposition as well as its $G$-ergodic one. Thus, in the case of $\mathscr{J}_{2}$, all our above conclusions may be extended from $G$ to $H$.

\section{Scaling Laws}

Assuming that the model has a critical point, characterised by the divergence of $l$, we now tentatively introduce a procedure designed to lead from the formalism of Section 4 to the so-called "scaling laws". These laws concern the asymptotic properties of the $\mu$-correlation function $F^{\prime}$ in the critical region. We shall formulate properties of this function, subject to certain specified assumptions, for cases where $g_{M}$, the interaction energy in a "hyperplane" $T$, is of the form

$$
g_{M}=g_{M}^{(e)}+h g_{M}^{(0)}
$$

where $g_{M}^{(e)}, g_{M}^{(0)}$ are even and odd functions, respectively, of $y_{M}$, and where $h$ is a constant. In the following analysis, we shall be concerned with the dependence of $F^{\prime}$ on $h$ and $\beta$. It should be noted that, in the particular case where $g_{M}^{(0)}=\sum_{t \in M} y_{t}$, the last term in Eq. (6.1) corresponds to the coupling of the spin system to an external magnetic field whose strength, in appropriate units, is $h$.

\footnotetext{
${ }^{9}$ For a definition of "amenable group", see Ref. [10].
} 
Let $\left\{E_{0}^{\prime}(\lambda)\right\}$ be the family of spectral projectors for $v_{0}^{\prime}$ (i.e. $\left.v_{0}^{\prime}=\int \lambda d E_{0}^{\prime}(\lambda)\right)$. Then it follows from Proposition 4.1 (iv) and Definition 4.6 that

$F^{\prime}\left(A^{\prime}, A ; n\right)=-\int_{-0}^{\lambda_{0}} \lambda^{n-n_{0}} d\left(\alpha^{*}\left(A^{\prime}\right),\left(E_{0}^{\prime}\left(\lambda_{0}\right)-E_{0}^{\prime}(\lambda)\right) \alpha(A)\right)_{\beta, h} ; \forall A, A^{\prime} \in \tilde{\mathscr{A}}$,
$n>n_{0}$,

where $\lambda_{0}=\exp \left(-l^{-1}\right)$,

and where the $(\beta, h)$ dependence of the inner product in Eq. (6.2) is indicated by the subscripts. On changing the variable of integration from $\lambda$ to $s\left(=\lambda / \lambda_{0}\right)$ in (6.2), we obtain the formula:

$F^{\prime}\left(A^{\prime}, A ; n\right)=-\exp -\left(\frac{n-n_{0}}{l}\right) \int_{-0}^{1} s^{n-n_{0}} d G_{A^{\prime} A}(\beta, h ; s), \forall A^{\prime}, A \in \tilde{\mathscr{A}}, n>n_{0}$,

where $\quad G_{A^{\prime} A}(\beta, h ; s)=\left(\alpha^{*}\left(A^{\prime}\right),\left(E_{0}^{\prime}\left(\lambda_{0}\right)-E_{0}^{\prime}\left(\lambda_{0} s\right)\right) \alpha(A)\right)_{\beta, h}$.

Noting that $l$ is a function of $\beta$ and $h$, we now introduce the following assumptions for $l$ and $G_{A^{\prime} A}$ :

(1) $\exists$ an inverse temperature $\beta_{c}$ such that $\lim _{\beta \rightarrow \beta_{c} \pm 0} \lim _{h \rightarrow 0} l(\beta, h)=0$; and further, $l_{A^{\prime} A}=l$ for the pair $\left(A^{\prime}, A\right)$ of elements of $\tilde{A}$ under consideration.

(2) $\exists$ a neighbourhood, $D$, of $\left(\beta_{c}, 0\right)$ in $R \times R$, and a real-valued function $\alpha$ on $D$ such that

(i) the map $\beta, h \rightarrow l, \alpha$ of $D$ onto $l(D) \times \alpha(D)$ is one-to-one,

(ii) $G_{A^{\prime} A}$ may be expressed in the form

$$
G_{A^{\prime} A}(\beta, h ; s)=(1-s)^{\nu(\alpha)} K_{A^{\prime} A}(l, \alpha ; s),
$$

where (iii) $v$ is a function from $\alpha(D)$ into $R_{+}$,

(iv) $K_{A^{\prime} A}(l, \alpha ; s)$ is finite for all $(l, \alpha, s) \in l(D) \times \alpha(D) \times[0,1]$, and is continuous with respect to $s$ at $s=1$,

(v) $\lim _{l \rightarrow \infty} K_{A^{\prime} A}(l, \alpha ; 1) \equiv k_{A^{\prime} A}(\alpha)$ exists and $\neq 0, \forall \alpha \in \alpha(D)$, and

(vi) $\frac{K_{A^{\prime} A}(l, \alpha ; 1)-K_{A^{\prime} A}(l, \alpha ; s)}{l(1-s)} \rightarrow 0$ as $l \rightarrow \infty$ for all $s \neq 1$,

the convergence being uniform with respect to $s$.

Note. Assumption (1) is designed to characterise the critical point $\left(\beta=\beta_{c}, h=0\right)$ in a manner that is consistent with the discussion of Section 5. Assumption 2 consists of a refined version of the statement that $G_{A^{\prime} A}$ behaves like a positive power of $(1-s)$ in the neighbourhood of $s=1$. 
Proposition 6.1. Let $f_{A^{\prime} A}(l, \alpha ; x) \equiv F^{\prime}\left(A^{\prime}, A ; n\right) l^{v(\alpha)}$ with $x=\frac{n}{l}$.

Then, under the assumptions (1) and (2),

$$
\lim _{l \rightarrow \infty} f_{A^{\prime} A}(l, \alpha ; x)=\frac{B_{A^{\prime} A}(\alpha) e^{-x}}{x^{\nu(\alpha)}},
$$

for fixed $x \neq 0$, with $B_{A^{\prime} A}(\alpha)=(2 \pi)^{1 / 2} \Gamma(v(\alpha)+1) k_{A^{\prime} A}(\alpha)$.

Note. This proposition corresponds to a statement of a generalised scaling law of the type proposed on phenomenological grounds by Coniglio and Marinaro [11].

Proof of Proposition 6.1. Since, by Eq. (6.5), $G_{A^{\prime} A}=0$ at $s=1$, it follows from the integration by parts of the integral in equation (6.4) that

$$
F^{\prime}\left(A^{\prime}, A ; n\right)=\left(n-n_{0}\right) \exp \left(-\frac{\left(n-n_{0}\right)}{l}\right) \int_{0}^{1} d s s^{n-n_{0}-1} G_{A^{\prime} A}(l, \alpha ; s) .
$$

Hence, by Eqs. (6.6) and (6.7), we may express $f_{A^{\prime} A}$ in the form

where

$$
f_{A^{\prime} A}=f_{A^{\prime} A}^{(1)}+f_{A^{\prime} A}^{(2)}
$$

$f_{A^{\prime} A}^{(1)}(l, \alpha ; x)=\left(x-\frac{n_{0}}{l}\right) \exp \left(\frac{n_{0}}{l}-x\right) l^{1+\nu(\alpha)} K_{A^{\prime} A}(l, \alpha ; 1) \int_{0}^{1} d s s^{l x-n_{0}-1}(1-s)^{\nu(\alpha)}$,

and

$$
\begin{aligned}
f_{A^{\prime} A}^{(2)}(l, \alpha ; x)= & \left(x-\frac{n_{0}}{l}\right) \exp \left(\frac{n_{0}}{l}-x\right) l^{2+v(\alpha)} \\
& \cdot \int_{0}^{1} d s s^{l x-n_{0}-1}(1-s)^{v(\alpha)+1}\left[\frac{K_{A^{\prime} A}(l, \alpha ; s)-K_{A^{\prime} A}(l, \alpha ; 1)}{l(1-s)}\right] .
\end{aligned}
$$

Now it follows from assumption $2(\mathrm{v})$ and Eq. (6.11) that, given $\varepsilon>0, \exists N(\varepsilon, \alpha)<\infty$ such that

$$
\begin{aligned}
\left|f_{A^{\prime} A}^{(2)}(l, \alpha ; x)\right| & <\varepsilon\left(x-\frac{n_{0}}{l}\right) \exp \left(\frac{n_{0}}{l}-x\right) l^{2+v(\alpha)} \\
& \cdot \int_{0}^{1} d s s^{l x-n_{0}-1}(1-s)^{v(\alpha)+1}, \quad \forall l>N(\varepsilon, \alpha) .
\end{aligned}
$$

Using the identity $\int_{0}^{1} d s s^{a-1}(1-s)^{b-1} \equiv \frac{\Gamma(a) \Gamma(b)}{(a+b)}$ for $a, b>0$, it follows from Eqs. (6.10), (6.12) that

$f_{A^{\prime} A}^{(1)}(l, \alpha ; x)=\left(x-\frac{n_{0}}{l}\right) \exp \left(\frac{n_{0}}{l}-x\right) K_{A^{\prime} A}(l, \alpha ; 1) \frac{\Gamma\left(l x-n_{0}\right) \Gamma(v(\alpha)+1)}{\Gamma\left(l x+v(\alpha)+1-n_{0}\right)}$ 
and

$$
\begin{aligned}
\left|f_{A^{\prime} A}^{(2)}(l, \alpha ; x)\right| & <\varepsilon\left(x-\frac{n_{0}}{l}\right) \exp \left(\frac{n_{0}}{l}-x\right) l^{2+v(\alpha)} \\
& \cdot \frac{\Gamma\left(l x-n_{0}\right) \Gamma(v(\alpha)+2)}{\Gamma\left(l x+v(\alpha)+2-n_{0}\right)}, \forall l>N(\varepsilon, \alpha) .
\end{aligned}
$$

Using the asymptotic formula $\Gamma(y) \approx(2 \pi)^{\frac{1}{2}} y^{y-\frac{1}{2}} e^{-y}$, it follows from assumption $2(\mathrm{v})$ and Eq. (6.13) that

$$
\lim _{l \rightarrow \infty} f_{A^{\prime} A}^{(1)}(l, \alpha ; x)=(2 \pi)^{\frac{1}{2}} \Gamma(v(\alpha)+1) k_{A^{\prime} A}(\alpha) e^{-x} / x^{v(\alpha)} .
$$

Similarly, it follows that, as $l \rightarrow \infty$, the coefficient of $\varepsilon$ on the right-hand side of $(6.14)$ tends to a finite value $\left(=(2 \pi)^{\frac{1}{2}} \Gamma(v(\alpha)+2) e^{-x} / x^{v(\alpha)+1}\right)$. Hence, the required result follows from Eqs. (6.13) and (6.15).

\section{Conclusion}

We have formulated spatial $(G-)$ correlations in Gibbs states of a specified class of Ising systems in terms of the quadruple $\left(\mathfrak{S}_{0}, \alpha, \alpha^{*}, v_{0}\right)$ (Propositions 4.1, 4.2). In particular, $G$-translations have been shown to correspond to the one-parameter semi-group $\left\{v_{0}^{n} \mid n \in Z_{+}\right\}$of contractions of $\mathfrak{H}_{0}$.

In cases (e.g. $\mathscr{J}_{2}$ ) where the model possesses the properties (a)-(d), specified in Section 5, the system has a critical point at the inverse temperature $\beta_{c}$, which is characterised by (A) a thermodynamical singularity, (B) a change in $\varrho$-symmetry associated with the $G$-ergodic decomposition of the Gibbs state, and (C) a divergence of the correlation length $l$ as $\beta \rightarrow \beta_{c} \pm 0$ (cf. discussion in Section 5). Further, the supplementary assumptions (1), (2) of Section 6 lead to a generalised scaling law (Proposition 6.1), of the type proposed on phenomenological grounds by Coniglio and Marinaro [11], in the critical region. Thus the formalism provides a framework for a systematic treatment of critical phenomena in Ising systems.

It is worth noting (cf. Section 5) that the characteristics (A), (B), (C) of the critical point depend on different features of $v_{0}$, and therefore their concurrence depends on some special properties of the model (e.g. (a)-(d)). In fact, there exist other models ${ }^{10}$, for which the critical point possesses properties (A) and (B), but not (C).

${ }^{10}$ For example, cf. the $B C S$ model as formulated by Thirring in terms of spins [16]. This model has a phase transition characterised by (A) and (B). However, since it corresponds to a mean-field theory, the correlation functions corresponding to its ergodic states may be easily shown to factorise in a manner which precludes the possibility of the characterisation $(\mathrm{C})$. 
Our formalism has some points of analogy with those employed for the treatment of a number of different models, in each of which spatial correlations were related to the spectral properties of a contraction of some Banach space (cf. Refs. $[6,12,13])$. Clearly, it would be of interest to have a basic theory, yielding conditions under which spatial correlations of a system correspond to a semi-group of such contractions.

\section{Appendix}

Proof of Lemma 3.1. It follows from Definitions 2.3 (i), 2.4 (i) and 3.1, together with the cyclicity of the $N$-boundary condition in Eq. (3.1), that (i) implies (ii). Further, by Definitions 2.3 (ii) and 3.1, together with the definitions of Cases (I), (II'), $\phi_{M N}=\phi_{M N^{\circ}} \varrho$ in those cases; and hence by Definition 2.4 (iii), (i) implies (iii). It therefore remains for us to prove (i). This we shall do firstly for Case (II), and then for Case (I).

Case (II). For each $L \in \mathscr{P}_{f}(S)$, we define $\sigma_{L}\left(\in \mathscr{A}_{L}\right)$ by the formula $\sigma_{L}(x)=\prod_{s \in L} x_{s}$. Then it follows from the generalised Griffiths inequalities (cf. [14]) that, for $L \subset M \times N, \phi_{M N}\left(\sigma_{L}\right)$ is a non-decreasing function of $M$ (i.e. $\phi_{M N}\left(\sigma_{L}\right) \geqq \phi_{M^{\prime} N}\left(\sigma_{L}\right)$ if $\left.M^{\prime} \subset M\right)$. Thus, since $\mathcal{N}-\lim _{N \rightarrow \infty} \phi_{M N}\left(\sigma_{L}\right)$ exists (by Eq. (4.22)) it follows that this limit is a bounded, non-decreasing function of $M$. Consequently $\mathscr{M}-\lim _{M \rightarrow \infty} \mathcal{N}-\lim _{N \rightarrow \infty} \phi_{M N}\left(\sigma_{L}\right)$ exists. for $\mathscr{A}$.

This establishes the required result, since $\left\{\sigma_{L} \mid L \in \mathscr{P}_{f}(S)\right\}$ is a basis

Case (I). In view of Eq. (4.24) (whose derivation does not depend on Lemma 3.1), it suffices for us to show that

$$
\mathscr{M}-\lim _{M \rightarrow \infty} \Psi_{M}(\hat{B}) \text { exists, } \forall \hat{B} \in \hat{\mathscr{B}}_{\mathscr{I}} .
$$

Let $M_{1}, M_{2}, M_{3}$ be arbitrary, mutually disjoint elements of $\mathscr{P}_{f}(T)$, and let $\hat{B}_{M_{1} M_{2} M_{3}}\left(\in \mathscr{\mathscr { B }}_{\mathscr{I}}\right)$ be defined by:

$$
\hat{B}_{M_{1} M_{2} M_{3}}=\prod_{t_{1} \in M_{1}} \hat{\sigma}_{t_{1}}^{(1)} \prod_{t_{2} \in M_{2}} \hat{\sigma}_{t_{2}}^{(2)} \prod_{t_{3} \in M_{3}} \hat{\sigma}_{t_{3}}^{(3)} .
$$

Then the set of all such $B_{M_{1} M_{2} M_{3}}$ 's form a basis for $\hat{\mathscr{B}}$, and thus (A.1) is equivalent to the condition that

$$
\mathscr{M}-\lim _{M \rightarrow \infty} \Psi_{M}\left(\hat{B}_{M_{1} M_{2} M_{3}}\right) \quad \text { exists }
$$

for all mutually disjoint $M_{1}, M_{2}, M_{3} \in \mathscr{P}_{f}(T)$.

Let $\hat{U}_{M}$ be the unitary, self-adjoint operator in $\mathscr{H}_{M}$ given by

24 Commun. math. Phys., Vol. 24

$$
\hat{U}_{M}=\prod_{t \in M} \hat{\sigma}_{t}^{(3)} .
$$


Then it follows from Eqs. (3.4), (4.3), (4.4) and (A.4), together with Definitions 2.6 and 4.2 (i), that $\hat{U}_{M}$ commutes with $\hat{v}_{M}$. Therefore, since the principal eigenvalue of $\hat{v}_{M}$ is non-degenerate it follows that $\hat{U}_{M} \hat{\Omega}_{M}$ $=\hat{\Omega}_{M} e^{i \gamma_{M}}$, where $\gamma_{M}$ is a phase angle; and thus, by Definition 4.2 (iii),

$$
\Psi_{M}\left(\hat{B}_{M_{1} M_{2} M_{3}}\right)=\Psi_{M}\left(\hat{U}_{M} \hat{B}_{M_{1} M_{2} M_{3}} \hat{U}_{M}^{-1}\right),
$$

for $M \supset M_{1} \cup M_{2} \cup M_{3}$. On the other hand, it follows from Eqs. (2.13), (A.2) and (A.4) that

$$
\hat{U}_{M} \hat{B}_{M_{1} M_{2} M_{3}} \hat{U}_{M}^{-1}=(-1)^{N_{12}} \hat{B}_{M_{1} M_{2} M_{3}},
$$

where $N_{12}$ is the number of sites in $M_{1} \cup M_{2}$. Consequently, by (A.5), $\Psi_{M}\left(\hat{B}_{M_{1} M_{2} M_{3}}\right)=0$ if $N_{12}$ is odd, in which case (A.3) is trivially satisfied.

It remains for us to establish (A.3) in the case where $N_{12}$ is even. For this purpose, we note that the SML techniques serve to express the properties of $\Psi_{M}$ in terms of those of an associated quasi-free state ${ }^{11}$ $\Psi_{M}^{\prime}$ on the algebra $\hat{\mathscr{B}}_{M}^{\prime}$ of CAR over $M$ : this is the algebra generated by a set $\left\{\alpha_{t}^{*}, \alpha_{t}\right\}$ such that $\left[\alpha_{t}, \alpha_{t^{\prime}}\right]_{+}=0$ and $\left[\alpha_{t}, \alpha_{t^{\prime}}^{*}\right]_{+}=\delta_{t t^{\prime}} I^{\prime}, \forall t, t^{\prime} \in M$.

It follows easily from SML that, if $N_{12}$ is even, $\Psi_{M}\left(\hat{B}_{M_{1} M_{2} M_{3}}\right)$ can be expressed in the form $\Psi_{M^{\prime}}^{\prime}\left(\hat{B}_{M_{1} M_{2} M_{3}}^{\prime}\right)$, where $\hat{B}_{M_{1} M_{2} M_{3}}^{\prime}$ is a uniquely defined $M$-independent element of $\hat{\mathscr{B}}^{\prime}{ }_{[a, b]}$, with a (resp. $b$ ) the least (resp. greatest) element $t$ in $M_{1} \cup M_{2} \cup M_{3}$. Consequently, in order to establish (A.3), it suffices for us to show that

$$
\mathscr{M}-\lim _{M \rightarrow \infty} \Psi_{M}^{\prime}\left(\hat{B}^{\prime}\right) \text { exists, } \quad \forall B^{\prime} \in \hat{\mathscr{B}}_{[a, b]}^{\prime} .
$$

Let $\hat{C}^{\prime}$ be an element of $\hat{\mathscr{B}}_{[a, b]}^{\prime}$ of the form $\hat{C}_{1}^{\prime} \hat{C}_{2}^{\prime} \ldots \hat{C}_{n}^{\prime}$, where each $\hat{C}_{r}^{\prime}$ is an element $\alpha_{t}$ or $\alpha_{t}^{*}$ of the set $\left\{\alpha_{t}, \alpha_{t}^{*} \mid t \in[a, b]\right\}$. Then, since $\Psi_{M}^{\prime}$ is a quasi-free state, it follows that $\Psi_{M}^{\prime}\left(\hat{C}^{\prime}\right)$ is a sum of products of terms $\Psi_{M}^{\prime}\left(\hat{C}_{r}^{\prime} \hat{C}_{s}^{\prime}\right)$. By a trivial extension of the analysis of SML (Section 4), one sees that $\Psi_{M}^{\prime}\left(\hat{C}_{r}^{\prime} \hat{C}_{s}^{\prime}\right)$, and thus $\Psi_{M}^{\prime}\left(\hat{C}^{\prime}\right)$, is $\mathscr{M}$-convergent as $M \rightarrow \infty$. Hence, since $\hat{\mathscr{B}}_{[a, b]}^{\prime}$ is the $C^{*}$-algebra generated by $\left\{\alpha_{t}, \alpha_{t}^{*} \mid t \in[a, b]\right\}$, it follows that (A.7) is valid.

Q.E.D.

\section{References}

1. Onsager, L.: Phys. Rev. 65, 117 (1944).

2. Montroll,E. W., Potts, R. B., Ward, J.C.: J. Math. Phys. 4, 308 (1963).

3. Kadanoff, L.P.: Physics 2, 263 (1966).

Kadanoff, L.P., et al.: Rev. Mod. Phys. 39, 395 (1967).

4. Dobrushin, R.L.: Theory Probability Appl. (USSR) (English Transl.) 13, 201 (1968);

Funct. Anal. Appl. 2, 31, 44 (1968).

5. Lanford, O., Ruelle, D.: J. Math. Phys. 8, 1460 (1967).

${ }^{11}$ For a definition of "quasi-free states", see for example, Ref. [15]. 
6. Kac, M.: Fundamental problems in statistical mechanics: II, pp. 71-105. Ed. E. G. D. Cohen. Amsterdam: North Holland Publ. 1968.

7. Schultz, T. D., Mattis, D. C., Lieb, E.: Rev. Mod. Phys. 36, 856 (1964).

8. Riesz, F., Sz.-Nagy, B.: Functional analysis. New York: Ungar 1955.

9. Emch, G.G., Knops, H.J.F., Verboven,E. J.: Commun. math. Phys. 8, 300 (1968).

10. Greenleaf,F.P.: Invariant means on topological groups. Princeton, N. J.: Van Nostrand, 1969.

11. Coniglio, A., Marinaro, M.: Physica 54, 261 (1971).

12. Ruelle, D.: Commun. math. Phys. 9, 267 (1967).

13. Araki, H.: Commun. math. Phys. 14, 120 (1967).

14. Ginibre, J.: Cargese Lectures, 1969.

15. Rocca, F., Sirugue, M., Testard, D.: Ann. Inst. H. Poincare 3, 247 (1969).

16. Thirring, W.: Commun. math. Phys. 7, 181 (1968).

M. Marinaro

G. L. Sewell

Dept. of Physics

Queen Mary College

London, E 1, England 
